\title{
Strong asymptotic freeness for Wigner and Wishart matrices
}

\author{
M. Capitaine* and C. Donati-Martin ${ }^{\dagger}$
}

\begin{abstract}
For each $n$ in $\mathbb{N}$, let $X_{n}=\left[\left(X_{n}\right)_{j k}\right]_{j, k=1}^{n}$ be a random Hermitian matrix such that the $n^{2}$ random variables $\sqrt{n}\left(X_{n}\right)_{i i}, \sqrt{2 n} R e\left(\left(X_{n}\right)_{i j}\right)_{i<j}$, $\sqrt{2 n} \operatorname{Im}\left(\left(X_{n}\right)_{i j}\right)_{i<j}$ are independent identically distributed with common distribution $\mu$ on $\mathbb{R}$. Let $X_{n}^{(1)}, \ldots, X_{n}^{(r)}$ be $r$ independent copies of $X_{n}$ and $\left(x_{1}, \ldots x_{r}\right)$ be a semicircular system in a $\mathcal{C}^{*}$-probability space. Assuming that $\mu$ is symmetric and satisfies a Poincaré inequality, we show that, almost everywhere, for any non commutative polynomial $p$ in $r$ variables,

$$
\lim _{n \longrightarrow+\infty}\left\|p\left(X_{n}^{(1)}, \ldots, X_{n}^{(r)}\right)\right\|=\left\|p\left(x_{1}, \ldots x_{r}\right)\right\| .
$$

We follow the method of [9] and [15] which gave (0.1) in the Gaussian (complex, real or symplectic) case. We also get that (0.1) remains true when the $X_{n}^{(i)}$ are Wishart matrices while the $x_{i}$ are Marchenko-Pastur distributed.
\end{abstract}

Mathematics Subject Classification (2000): 15A52, 46L54, 60F99.

Key words: Random matrices, free probability, asymptotic freeness.

*Laboratoire de Statistique et Probabilités, Université Paul Sabatier, 118 route de Narbonne, F-31062 Toulouse Cedex. E-mail: capitain@cict.fr

${ }^{\dagger}$ Laboratoire de Probabilités et Modèles Aléatoires, Université Paris 6, Site Chevaleret, 13 rue Clisson, F-75013 Paris. E-mail: donati@ccr.jussieu.fr 


\section{Introduction}

In the 90's, Voiculescu [18] introduced a random matrix model for a free semicircular system. He showed that if we take $r$ independent random matrices $\left(X_{n}^{(i)}\right)_{i=1, \ldots .}$, distributed as $G U E\left(n, \frac{1}{n}\right)$, then, they are asymptotically free, that is, for every non commutative polynomial $p$ in $r$ variables,

$$
\mathbb{E}\left[\operatorname{tr}_{n} p\left(X_{n}^{(1)}, \ldots, X_{n}^{(r)}\right)\right] \underset{n \longrightarrow \infty}{\longrightarrow} \tau\left(p\left(x_{1}, \ldots x_{r}\right)\right)
$$

where $t r_{n}$ stands for the normalized trace on $M_{n}(\mathbb{C})$ and $\left(x_{1}, \ldots x_{r}\right)$ is a free family of semicircular variables in some non commutative probability space $(\mathcal{B}, \tau)$. The result (1.1) holds true for a family of iid Wigner matrices and is proved by Dykema in [6].

In a recent paper, Haagerup and Thorbjørnsen [9] proved a strong version of (1.1), in the GUE case, namely a convergence for the operator norm:

$$
\lim _{n \longrightarrow+\infty}\left\|p\left(X_{n}^{(1)}, \ldots, X_{n}^{(r)}\right)\right\|=\left\|p\left(x_{1}, \ldots x_{r}\right)\right\| \text { a.s. }
$$

which led to the proof that $\operatorname{Ext}\left(C_{r e d}^{*}\left(F_{2}\right)\right)$ is not a group.

Schultz [15] obtained the same result for Gaussian random matrices in the real case (GOE) and in the simplectic case (GSE). Our aim is to extend (1.2) in the case of an independent family of Wigner matrices on one hand and in the case of Wishart matrices on the other hand . Note that the special case $r=1$ gives the well known convergence of the largest eigenvalue of $X_{n}^{(1)}$ to the right boundary of the support of $x_{1}$ (see [3] for the Wigner case and [7] for the Wishart case; see also [2] and the references therein).

Our approach is very similar to that of [9] and [15]. Therefore, we will recall the main lines of their proofs. First, in proving (1.2), the minoration

$$
\liminf _{n \longrightarrow+\infty}\left\|p\left(X_{n}^{(1)}, \ldots, X_{n}^{(r)}\right)\right\| \geq\left\|p\left(x_{1}, \ldots x_{r}\right)\right\| \text { a.s. }
$$

comes rather easily from an a.s. version of (1.1) (obtained in [17] for the GUE case and proved in Section 6 of [15] for the GOE case) (see Lemma 7.2 in [9]). So, the main difficulty is the proof of the reverse inequality:

$$
\limsup _{n \longrightarrow+\infty}\left\|p\left(X_{n}^{(1)}, \ldots, X_{n}^{(r)}\right)\right\| \leq\left\|p\left(x_{1}, \ldots x_{r}\right)\right\| \text { a.s. }
$$

In the following, we sketch the main steps in the proof of (1.3).

Step 1: A linearisation trick (see 9], Section 2 and Proposition 7.3) In order to prove (1.3), it is sufficient to prove: 
Lemma 1.1 For all $m \in \mathbb{N}$, all self-adjoint matrices $a_{0}, \ldots, a_{r}{ }^{1}$ of size $m \times m$ and all $\epsilon>0$,

$$
\left.s p\left(a_{0} \otimes 1_{n}+\sum_{i=1}^{r} a_{i} \otimes X_{n}^{(i)}(\omega)\right) \subset \operatorname{sp}\left(a_{0} \otimes 1_{\mathcal{B}}+\sum_{i=1}^{r} a_{i} \otimes x_{i}\right)+\right]-\epsilon, \epsilon[
$$

eventually, as $n \longrightarrow \infty$ a.e. in $\omega$. Here, $s p(T)$ denotes the spectrum of the operator $T$ and $1_{n}$ the identity matrix.

The analysis of the spectrum of $S_{n}:=a_{0} \otimes 1_{n}+\sum_{i=1}^{r} a_{i} \otimes X_{n}^{(i)}$ is done, using the Stieljes transform

$G_{n}(\lambda)=\mathbb{E}\left[\left(i d_{m} \otimes \operatorname{tr}_{n}\right)\left[\left(\lambda \otimes 1_{n}-S_{n}\right)^{-1}\right]\right], \lambda \in M_{m}(\mathbb{C}), \operatorname{Im}(\lambda)$ positive definite.

The proof of (1.4) requires sharp estimates of the rate of convergence of $G_{n}(\lambda)$ to $G(\lambda):=\left(i d_{m} \otimes \tau\right)\left[\left(\lambda \otimes 1_{\mathcal{B}}-s\right)^{-1}\right]$ (of order $\left.1 / n^{2}\right)$ where $s=$ $a_{0} \otimes 1_{\mathcal{B}}+\sum_{p=1}^{r} a_{p} \otimes x_{p}$

Step 2: In the GUE case, Haagerup and Thorbjørnsen [9] obtains the following estimate

$$
\left\|G_{n}(\lambda)-G(\lambda)\right\| \leq \frac{C(\lambda)}{n^{2}} .
$$

In the GOE, GSE cases, Schultz [15] gets an extra term of order $1 / n$, namely

$$
\left\|G_{n}(\lambda)-G(\lambda)-\frac{L(\lambda)}{n}\right\| \leq \frac{C(\lambda)}{n^{2}}
$$

for some functional $L$.

Step 3 From the previous step, it is shown in section 6 of 9 ] that

$$
\mathbb{E}\left[\left(\operatorname{tr}_{m} \otimes \operatorname{tr}_{n}\right)\left(\varphi\left(S_{n}\right)\right)\right]=\left(\operatorname{tr}_{m} \otimes \tau\right)(\varphi(s))+O\left(\frac{1}{n^{2}}\right)
$$

for $\varphi$ smooth with compact support, and

$$
\mathbb{E}\left[\left(\operatorname{tr}_{m} \otimes \operatorname{tr}_{n}\right)\left(\varphi\left(S_{n}\right)\right)\right]=O\left(\frac{1}{n^{2}}\right)
$$

for $\varphi$ smooth, constant outside a compact set and such that $\operatorname{supp}(\varphi) \cap \operatorname{sp}(s)=$ $\emptyset$. In the GOE case (resp. GSE case), Schultz proved in section 5 of [15] that

$$
\mathbb{E}\left[\left(\operatorname{tr}_{m} \otimes \operatorname{tr}_{n}\right)\left(\varphi\left(S_{n}\right)\right)\right]=\left(\operatorname{tr}_{m} \otimes \tau\right)(\varphi(s))+\frac{1}{n} \Lambda(\varphi)+O\left(\frac{1}{n^{2}}\right)
$$

\footnotetext{
${ }^{1}$ By a density argument, we can also assume that the matrices $a_{i}$ are invertible.
} 
where $\Lambda$ is a distribution with compact support in $s p(s)$ with Stieljes transform

$$
f(\lambda)=\operatorname{tr}_{m}\left(L\left(\lambda 1_{m}\right)\right), \lambda \in \mathbb{C} \backslash \mathbb{R} .
$$

Therefore, (1.9) still holds for $\varphi$ with $\operatorname{supp}(\varphi) \cap s p(s)=\emptyset$.

Step 4 (1.9), combining with a Gaussian variance estimate, yields (by a standard application of the Borel Cantelli lemma),

$$
\left(\operatorname{tr}_{m} \otimes \operatorname{tr}_{n}\right) 1_{F}\left(S_{n}\right)=O\left(n^{-4 / 3}\right)
$$

for $F=\{t \in \mathbb{R}, d(t, s p(s)) \geq \epsilon\}$ which leads to (1.4).

The main difficulties in the generalization of the above to Wigner or Wishart matrices arise in step 2. Indeed, we don't have the gaussian integration by parts' formula anymore. Our approach is inspired by the work of [12] where they use a Taylor expansion (see Lemma 4.1) extending the gausian integration by parts' formula. The remainder of the proof can be completed essentially as in the GOE/GSE case. Hence, in this paper, we shall focus on the obtention of such a master inequality

$$
\left\|G_{n}(\lambda)-G(\lambda)-\frac{1}{n} L(\lambda)\right\|=O\left(\frac{1}{n^{2}}\right)
$$

in the case of a family of Hermitian matrices with symmetric iid entries satisfying a Poincaré inequality, as well as in the case of Wishart matrices; we just give some hints when the computations are similar to that of [9, [15.

The paper is organized as follows. In section 2, we introduce notations and preliminaries which will be of basic use later on. In section 3, we describe the proof of (1.8) and (1.10) proved respectively in [9] and [15] in order to make clear the validity of the method in our general framework we state in section 4 (for the Wigner case) and section 5 (for the Wishart case).

\section{Notations and preliminaries}

This section may contain some definitions already used in the introduction but we choose to gather all the notations in this section for the reader's convenience. To begin with, we introduce some notations on the set of matrices. 
- $M_{p}(\mathbb{C})$ is the set of $p \times p$ matrices with complex entries, $M_{p}(\mathbb{C})_{s a}$ the subset of self-adjoint elements of $M_{p}(\mathbb{C})$ and $1_{p}$ the identity matrix. In the following, we shall consider two sets of matrices with $p=m(m$ fixed) and $p=n$ with $n \longrightarrow \infty$.

- $\operatorname{Tr}_{p}$ denotes the trace and $\operatorname{tr}_{p}=\frac{1}{p} \operatorname{Tr}_{p}$ the normalized trace on $M_{p}(\mathbb{C})$.

- $\|$.$\| denotes the operator norm on M_{p}(\mathbb{C})$ and $\|M\|_{2}=\left(\operatorname{Tr}_{p}\left(M^{*} M\right)\right)^{1 / 2}$ the Hilbert-Schmidt norm.

- Let $\left(E_{i j}\right)_{i, j=1}^{n}$ be the canonical basis of $M_{n}(\mathbb{C})$ and define a basis of the real vector space of the self-adjoint matrices $M_{n}(\mathbb{C})_{s a}$ by:

$$
\begin{aligned}
e_{j j} & =E_{j j}, 1 \leq j \leq n \\
e_{j k} & =\frac{1}{\sqrt{2}}\left(E_{j k}+E_{k j}\right), 1 \leq j<k \leq n \\
f_{j k} & =\frac{i}{\sqrt{2}}\left(E_{j k}-E_{k j}\right), 1 \leq j<k \leq n
\end{aligned}
$$

- For a matrix $M$ in $M_{m}(\mathbb{C}) \otimes M_{n}(\mathbb{C})$, we denote by

$$
M_{i j}:=\left(i d_{m} \otimes \operatorname{Tr}_{n}\right)\left(M\left(1_{m} \otimes E_{j i}\right)\right) \in M_{m}(\mathbb{C}), 1 \leq i, j \leq n
$$

and

$$
{ }_{\alpha, \beta} M:=\left(\operatorname{Tr}_{m} \otimes i d_{n}\right)\left(M\left(\hat{E}_{\beta, \alpha} \otimes 1_{n}\right)\right) \in M_{n}(\mathbb{C}), 1 \leq \alpha, \beta \leq m
$$

where $\left(\hat{E}_{\alpha, \beta}\right)$ is the canonical basis of $M_{m}(\mathbb{C})$.

We now define our matrix model and the random variables of interest.

- $\left(X_{n}^{(1)}, \ldots, X_{n}^{(r)}\right)_{i=1, \ldots . r}$ is a set of iid random matrices in $M_{n}(\mathbb{C})_{s a}$, whose distribution will be specified later (matrices in GUE or GOE in section 3, Wigner matrices in Section 4, Wishart matrices in section 5).

- For a given family $a_{0}, \ldots a_{r}$ in $M_{m}(\mathbb{C})_{s a}$, we define the random variable $S_{n}$ with values in $M_{m}(\mathbb{C}) \otimes M_{n}(\mathbb{C})$ by:

$$
S_{n}=a_{0} \otimes 1_{n}+\sum_{p=1}^{r} a_{p} \otimes X_{n}^{(p)}
$$


and $s \in M_{m}(\mathbb{C}) \otimes \mathcal{B}$ by

$$
s=a_{0} \otimes 1_{\mathcal{B}}+\sum_{p=1}^{r} a_{p} \otimes x_{p}
$$

where the $\left(x_{i}\right)_{i=1, \ldots r}$ is a free family of self-adjoint operators in a $C^{*}$ probability space $(\mathcal{B}, \tau)$ with a faithful state $\tau$, whose distribution will be specified in the different cases (semi-circular in sections 3 and 4 or distributed as the Marchenko-Pastur distribution in section 5).

- For any matrix $\lambda$ in $\mathcal{O}$ where

$$
\mathcal{O}:=\left\{\lambda \in M_{m}(\mathbb{C}) \mid \operatorname{Im}(\lambda) \text { is positive definite }\right\},
$$

we define the $M_{m}(\mathbb{C})$ valued rv:

$$
\begin{gathered}
H_{n}(\lambda)=\left(i d_{m} \otimes \operatorname{tr}_{n}\right)\left[\left(\lambda \otimes 1_{n}-S_{n}\right)^{-1}\right], \\
G_{n}(\lambda)=\mathbb{E}\left[H_{n}(\lambda)\right]
\end{gathered}
$$

and

$$
G(\lambda)=\left(i d_{m} \otimes \tau\right)\left[\left(\lambda \otimes 1_{\mathcal{B}}-s\right)^{-1}\right] .
$$

For $\lambda \in \mathbb{C} \backslash \mathbb{R}$, we also define

$$
g_{n}(\lambda)=\operatorname{tr}_{m}\left(G_{n}\left(\lambda 1_{m}\right)\right)
$$

and

$$
g(\lambda)=\operatorname{tr}_{m}\left(G\left(\lambda 1_{m}\right)\right) .
$$

We end this preliminary by recalling some properties of $G(\lambda)$ and of the resolvent $\left(\lambda \otimes 1_{n}-S_{n}\right)^{-1}$ of the matrix $S_{n}$. First, one can easily see that for any $\lambda$ and $\lambda^{\prime}$ in $M_{m}(\mathbb{C})$ such that $\operatorname{Im}(\lambda)$ and $\operatorname{Im}\left(\lambda^{\prime}\right)$ are positive definite,

$$
\left(\lambda \otimes 1_{\mathcal{B}}-s\right)^{-1}-\left(\lambda^{\prime} \otimes 1_{\mathcal{B}}-s\right)^{-1}=\left(\lambda \otimes 1_{\mathcal{B}}-s\right)^{-1}\left(\lambda^{\prime}-\lambda\right)\left(\lambda^{\prime} \otimes 1_{\mathcal{B}}-s\right)^{-1} .
$$

Lemma 2.1 Let $\lambda$ in $M_{m}(\mathbb{C})$ such that $\operatorname{Im}(\lambda)$ is positive definite. Then

$$
\left\|\left(\lambda \otimes 1_{\mathcal{B}}-s\right)^{-1}\right\| \leq\left\|\operatorname{Im}(\lambda)^{-1}\right\| \quad \text { and } \quad\|G(\lambda)\| \leq\left\|\operatorname{Im}(\lambda)^{-1}\right\| .
$$

Moreover, $G(\lambda)$ is invertible and

$$
\left\|G(\lambda)^{-1}\right\| \leq(\|\lambda\|+\|s\|)^{2}\left\|\operatorname{Im}(\lambda)^{-1}\right\| .
$$


We refer the reader to section 5 of [9] for a proof of (2.8).

Lemma 2.2 Let $\lambda$ in $M_{m}(\mathbb{C})$ such that $\operatorname{Im}(\lambda)$ is positive definite, then

$$
\begin{gathered}
\left\|\left(\lambda \otimes 1_{n}-S_{n}\right)^{-1}\right\| \leq\left\|\operatorname{Im}(\lambda)^{-1}\right\|, \\
\forall 1 \leq k, l \leq n,\left\|\left(\lambda \otimes 1_{n}-S_{n}\right)_{k l}^{-1}\right\| \leq\left\|\operatorname{Im}(\lambda)^{-1}\right\|,
\end{gathered}
$$

and for $p \geq 2$,

$$
\frac{1}{n} \sum_{k, l=1}^{n}\left\|\left(\lambda \otimes 1_{n}-S_{n}\right)_{k l}^{-1}\right\|^{p} \leq C_{m}\left\|\operatorname{Im}(\lambda)^{-1}\right\|^{p}
$$

where, in the first inequality, $\|$.$\| denotes the operator norm in M_{m}(\mathbb{C}) \otimes$ $M_{n}(\mathbb{C})$ ( in $M_{m}(\mathbb{C})$ in the others) and $C_{m}$ a constant depending only on $m$.

For a Hermitian matrix $M$, the derivative w.r.t $M$ of the resolvent $R(z)=$ $(z-M)^{-1}$ satisfies:

$$
R_{M}^{\prime}(z) . A=R(z) A R(z) \text { for all Hermitian matrix } A \text {. }
$$

Sketch of Proof: We just mention the proof of (2.11). From (2.10), it's enough to consider the case $p=2$.

Let us denote $G^{(n)}=\left(\lambda \otimes 1_{n}-S_{n}\right)^{-1} \in M_{m}(\mathbb{C}) \otimes M_{n}(\mathbb{C})$. Since the operator norm is smaller than the Hilbert-Schmidt norm,

$$
\begin{aligned}
\frac{1}{n} \sum_{k, l=1}^{n}\left\|G_{k l}^{(n)}\right\|^{2} & \leq\left.\left.\frac{1}{n} \sum_{k, l=1}^{n} \sum_{\alpha, \beta=1}^{m}\right|_{\alpha, \beta} G_{k l}^{(n)}\right|^{2} \\
& =\frac{1}{n} \operatorname{Tr}_{n m}\left(G^{(n)}\left(G^{(n)}\right)^{*}\right) \\
& \leq m\left\|G^{(n)}\left(G^{(n)}\right)^{*}\right\| \leq m\left\|\operatorname{Im}(\lambda)^{-1}\right\|^{2} .
\end{aligned}
$$

where the last inequality follows from (2.9).

In the sequel, we shall denote by $P_{k}$ any polynomial of degree $k$ whose coefficients are positive and by $C$ or $K$ any constant; $P_{k}, C$ or $K$ can depend on the $a_{l}, l=1, \ldots, r$, and may vary from line to line. 


\section{Main ideas in the proofs of (1.8) and (1.10) from [9] and [15]}

\subsection{Estimate of $\left\|G_{n}-G\right\|$ in [9]}

Let us recall the main ideas of [9] in the estimation of $\left\|G_{n}(\lambda)-G(\lambda)\right\|$. In lemma 5.4 of [9], Haagerup and Thorbjørnsen observe in one hand that the matrix-valued Stieljes transform of $s$ satisfies, for any $\lambda$ in $\mathcal{O}$,

$$
\sum_{i=1}^{r} a_{i} G(\lambda) a_{i}+\left(a_{0}-\lambda\right)+G(\lambda)^{-1}=0
$$

In the other hand, using the Gaussian integration by parts formula, they establish the analogue of (3.1) satisfied by $H_{n}(\lambda)$ ("Master equation", Lemma 2 [9]):

$$
\mathbb{E}\left[\sum_{p=1}^{r} a_{p} H_{n}(\lambda) a_{p} H_{n}(\lambda)+\left(a_{0}-\lambda\right) H_{n}(\lambda)+1_{m}\right]=0 .
$$

Then, using the Gaussian Poincaré inequality to get an estimate of the variance of $H_{n}(\lambda)$, they deduce from (3.2) the "Master inequality" (Lemma 3 in 9]):

$$
\left\|\sum_{i=1}^{r} a_{i} G_{n}(\lambda) a_{i} G_{n}(\lambda)+\left(a_{0}-\lambda\right) G(\lambda)+1_{m}\right\| \leq \frac{C}{n^{2}}\left\|\operatorname{Im}(\lambda)^{-1}\right\|^{4} .
$$

Moreover, the authors prove that $G_{n}(\lambda)$ is invertible for any $\lambda$ in $\mathcal{O}$ and they give an upper bound of the norm of its inverse (Proposition 5.2 [9])

$$
\left\|G_{n}(\lambda)^{-1}\right\| \leq(\|\lambda\|+K)^{2}\left\|\operatorname{Im}(\lambda)^{-1}\right\| .
$$

Hence, they deduce from (3.3) that, for any $\lambda$ in $\mathcal{O}$,

$$
\left\|a_{0}+\sum_{i=1}^{r} a_{i} G_{n}(\lambda) a_{i}+G_{n}(\lambda)^{-1}-\lambda\right\| \leq f_{n}\left(\left\|\operatorname{Im}(\lambda)^{-1}\right\|,\|\lambda\|\right),
$$

where

$$
f_{n}\left(\left\|\operatorname{Im}(\lambda)^{-1}\right\|,\|\lambda\|\right)=\frac{C}{n^{2}}(\|\lambda\|+K)^{2}\left\|\operatorname{Im}(\lambda)^{-1}\right\|^{5} .
$$


Further, they set

$$
\Lambda_{n}(\lambda)=a_{0}+\sum_{i=1}^{r} a_{i} G_{n}(\lambda) a_{i}+G_{n}(\lambda)^{-1}
$$

for any $\lambda$ in $\mathcal{O}$. (3.4) can be rewritten

$$
\left\|\Lambda_{n}(\lambda)-\lambda\right\| \leq f_{n}\left(\left\|\operatorname{Im}(\lambda)^{-1}\right\|,\|\lambda\|\right) .
$$

The authors define

$$
\mathcal{O}_{n}^{\prime}=\left\{\lambda \in \mathcal{O}, f_{n}\left(\left\|\operatorname{Im}(\lambda)^{-1}\right\|,\|\lambda\|\right)<\frac{\epsilon(\lambda)}{2}\right\}
$$

where

$$
\epsilon(\lambda):=\frac{1}{\left\|\operatorname{Im}(\lambda)^{-1}\right\|} .
$$

(3.5) implies that, for any $\lambda$ in $\mathcal{O}_{n}^{\prime}$,

$$
\operatorname{Im} \Lambda_{n}(\lambda) \geq \frac{1}{2\left\|\operatorname{Im}(\lambda)^{-1}\right\|} 1_{m}
$$

and that in particular $\Lambda_{n}(\lambda)$ belongs to $\mathcal{O}$ (see Lemma 5.5 [9]). Consequently, applying (3.1), they get that, for any $\lambda$ in $\mathcal{O}_{n}^{\prime}$,

$$
a_{0}+\sum_{i=1}^{r} a_{i} G\left(\Lambda_{n}(\lambda)\right) a_{i}+G\left(\Lambda_{n}(\lambda)\right)^{-1}=a_{0}+\sum_{i=1}^{r} a_{i} G_{n}(\lambda) a_{i}+G_{n}(\lambda)^{-1}
$$

In proof of (b) Proposition 5.6, Haagerup and Thorbjørnsen show that (3.7) implies that

$$
G_{n}(\lambda)=G\left(\Lambda_{n}(\lambda)\right)
$$

for any $\lambda$ in $\mathcal{O}_{n}^{\prime \prime}:=\left\{\lambda \in \mathcal{O}_{n}^{\prime}, \epsilon(\lambda)>\sqrt{2 \sum_{i=1}^{r}\left\|a_{i}\right\|^{2}}\right\}$. Using that $t \mapsto$ $f_{n}\left(t^{-1}, t\right) t^{-1}$ is a continuous strictly decreasing function from ]0; $+\infty$ [ onto ]0; $+\infty$ [, they show in proof of (a) Proposition 5.6 [9] that $\mathcal{O}_{n}^{\prime}$ is an open connected subset of $M_{m}(\mathbb{C})$. Thus, by the principle of uniqueness of analytic continuation, (3.8) still holds for any $\lambda$ in $\mathcal{O}_{n}^{\prime}$. Thus, for any $\lambda$ in $\mathcal{O}_{n}^{\prime}$, they get that

$$
\begin{aligned}
\left\|G_{n}(\lambda)-G(\lambda)\right\| & \leq\left\|G\left(\Lambda_{n}(\lambda)\right)-G(\lambda)\right\| \\
& \leq\left\|\operatorname{Im}\left(\Lambda_{n}(\lambda)\right)^{-1}\right\|\left\|\lambda-\Lambda_{n}(\lambda)\right\|\left\|\operatorname{Im}(\lambda)^{-1}\right\| \\
& \leq 2 f_{n}\left(\left\|\operatorname{Im}(\lambda)^{-1}\right\|,\|\lambda\|\right)\left\|\operatorname{Im}(\lambda)^{-1}\right\|^{2}
\end{aligned}
$$


where the last inequality comes from (3.5), (3.6). Now, if $\lambda$ belongs to $\mathcal{O} \backslash \mathcal{O}_{n}^{\prime}$, they note that

$$
\begin{aligned}
\left\|G_{n}(\lambda)-G(\lambda)\right\| & \leq 2\left\|\operatorname{Im}(\lambda)^{-1}\right\| \\
& \leq 4 f_{n}\left(\left\|\operatorname{Im}(\lambda)^{-1}\right\|,\|\lambda\|\right)\left\|\operatorname{Im}(\lambda)^{-1}\right\|^{2}
\end{aligned}
$$

since

$$
\frac{1}{2} \leq f_{n}\left(\left\|\operatorname{Im}(\lambda)^{-1}\right\|,\|\lambda\|\right)\left\|\operatorname{Im}(\lambda)^{-1}\right\| .
$$

Finally, for any $\lambda$ in $\mathcal{O}$,

$$
\begin{aligned}
\left\|G_{n}(\lambda)-G(\lambda)\right\| & \leq 4 f_{n}\left(\left\|\operatorname{Im}(\lambda)^{-1}\right\|,\|\lambda\|\right)\left\|\operatorname{Im}(\lambda)^{-1}\right\|^{2} \\
& =\frac{C}{n^{2}}(\|\lambda\|+K)^{2}\left\|\operatorname{Im}(\lambda)^{-1}\right\|^{7}
\end{aligned}
$$

\subsection{Estimate of $\left\|G_{n}-G-\frac{1}{n} L\right\|$ in [15]}

In the GOE case, a term of order $1 / n$ appears in the Master equation so that the estimate of $\left\|G_{n}(\lambda)-G(\lambda)\right\|$ Schultz makes by sticking to the previous proof of [9] is of order $1 / n$. Nevertheless, a further study (we will describe in our general framework in section 4) gives her the sharper estimate

$$
\left\|G_{n}(\lambda)-G(\lambda)-\frac{1}{n} L(\lambda)\right\| \leq \frac{1}{n^{2}}(\|\lambda\|+K)^{8} P_{13}\left(\left\|\operatorname{Im}(\lambda)^{-1}\right\|\right)
$$

for any $\lambda$ such that $\operatorname{Im} \lambda$ positive definite or negative definite.

\subsection{From Step 2 to Step 3}

¿From the previous estimates (3.9) and (3.10), Haagerup, Thorbjørnsen and Schultz immediately get that, for any $\lambda$ in $\mathbb{C} \backslash \mathbb{R}$,

$$
\left|r_{n}(\lambda)\right| \leq \frac{1}{n^{2}}(\|\lambda\|+K)^{\alpha} P_{k}\left(\left|\operatorname{Im}(\lambda)^{-1}\right|\right)
$$

where

-in the GUE case [9]

$$
r_{n}(\lambda)=g_{n}(\lambda)-g(\lambda), \alpha=2 k=7
$$


-in the GOE case [15]

$$
r_{n}(\lambda)=g_{n}(\lambda)-g(\lambda)-\frac{1}{n} t r_{m}\left(L\left(\lambda 1_{m}\right)\right), \alpha=8 k=13 .
$$

Since $S_{n}$ and $s$ are selfadjoint, by the spectral theory, there exist unique probability measures $\mu_{n}$ and $\mu$ on $\mathbb{R}$ such that

$$
\begin{gathered}
\int \varphi d \mu_{n}=\mathbb{E}\left[\left(\operatorname{tr}_{m} \otimes \operatorname{tr}_{n}\right)\left(\varphi\left(S_{n}\right)\right)\right] \\
\int \varphi d \mu=\left(\operatorname{tr}_{m} \otimes \tau\right)(\varphi(s)) .
\end{gathered}
$$

$g_{n}$ and $g$ are the Stieljes transforms of $\mu_{n}$ and $\mu$. Moreover, in Lemma 5.5 in 15, Schultz proves by using a characterisation theorem of Tillmann that $l(\lambda):=\operatorname{tr}_{m}\left(L\left(\lambda 1_{m}\right)\right)$ is the Stieljes transform of a distribution $\Lambda$ with compact support in $s p(s)$. Hence, using the inverse Stieljes tranform, Haagerup, Thorbjornsen and Schultz get respectively that, for any $\varphi$ in $\mathcal{C}_{c}^{\infty}(\mathbb{R}, \mathbb{R})$,

- in 9

$$
\int \varphi d \mu_{n}-\int \varphi d \mu=-\frac{1}{\pi} \lim _{y \rightarrow 0^{+}} \operatorname{Im} \int_{\mathbb{R}} \varphi(x) r_{n}(x+i y) d x .
$$

- in 15

$$
\int \varphi d \mu_{n}-\int \varphi d \mu-\frac{\Lambda(\varphi)}{n}=-\frac{1}{\pi} \lim _{y \rightarrow 0^{+}} \operatorname{Im} \int_{\mathbb{R}} \varphi(x) r_{n}(x+i y) d x .
$$

Hence, the remainder of the two proofs (in [9] and [15]) deals with the estimation of

$$
\limsup _{y \rightarrow 0^{+}}\left|\int_{\mathbb{R}} \varphi(x) h(x+i y) d x\right|
$$

where $h$ is an analytic function on $\mathbb{C} \backslash \mathbb{R}$ which satisfies

$$
|h(\lambda)| \leq(\|\lambda\|+K)^{\alpha} P_{k}\left(\left|\operatorname{Im}(\lambda)^{-1}\right|\right) .
$$

In 9] section 6, Haagerup and Thorbjørnsen introduce a very clever family of functions $\left\{I_{p}(\lambda), p \geq 1\right\}$ defined by

$$
I_{p}(\lambda)=\frac{1}{(p-1) !} \int_{0}^{+\infty} h(\lambda+t) t^{p-1} \exp (-t) d t .
$$


They note that

$$
\begin{gathered}
I_{1}(\lambda)-I_{1}^{\prime}(\lambda)=h(\lambda) \\
I_{p}(\lambda)-I_{p}^{\prime}(\lambda)=I_{p-1}(\lambda), p \geq 2,
\end{gathered}
$$

so that for any $\varphi$ in $\mathcal{C}_{c}^{\infty}(\mathbb{R}, \mathbb{R})$ and $y>0$,

$$
\int_{\mathbb{R}} \varphi(x) h(x+i y) d x=\int_{\mathbb{R}}(1+D)^{p} \varphi(x) I_{p}(x+i y) d x .
$$

Now, they choose $p=k+1$ where $k$ is the degree of the polynomial in the right hand side of (3.14) (that is $p=8$ in [9] and $p=14$ in [15]) and estimate $I_{k+1}(\lambda)$ for $\operatorname{Im} \lambda>0$. Using (3.14), it is not difficult to see that

$$
\lim _{r \rightarrow+\infty} \int_{[r, r+i r]} \frac{1}{k !} h(\lambda+z) z^{k} \exp (-z) d z=0 .
$$

Thus, by Cauchy's integral theorem, the authors get

$$
\begin{aligned}
I_{k+1}(\lambda) & =\lim _{r \rightarrow+\infty} \int_{[0, r+i r]} \frac{1}{k !} h(\lambda+z) z^{k} \exp (-z) d z \\
& =\int_{0}^{+\infty} \frac{1}{k !} h(\lambda+(1+i) t)(1+i)^{k+1} t^{k} \exp (-(1+i) t) d t .
\end{aligned}
$$

Plugging in (3.14), one gets for any $\lambda$ such that $\operatorname{Im} \lambda>0$,

$$
\begin{aligned}
\left|I_{k+1}(\lambda)\right| & \leq \frac{2^{\frac{k+1}{2}}}{k !} \int_{0}^{+\infty}(|\lambda|+\sqrt{2} t+K)^{\alpha} P_{k}\left(|\operatorname{Im} \lambda+t|^{-1}\right) t^{k} \exp (-t) d t \\
& \leq \frac{2^{\frac{k+1}{2}}}{k !} \int_{0}^{+\infty}(|\lambda|+\sqrt{2} t+K)^{\alpha} P_{k}\left(t^{-1}\right) t^{k} \exp (-t) d t \\
& \leq \int_{0}^{+\infty}(|\lambda|+\sqrt{2} t+K)^{\alpha} Q(t) \exp (-t) d t
\end{aligned}
$$

where $Q(t)=\frac{2^{\frac{k+1}{2}}}{k !} P_{k}\left(t^{-1}\right) t^{k}$ is a polynomial.

It follows by dominated convergence

$\limsup _{y \rightarrow 0^{+}}\left|\int_{\mathbb{R}} \varphi(x) h(x+i y) d x\right| \leq \int_{\mathbb{R}} \int_{0}^{+\infty}\left|(1+D)^{p} \varphi(x)\right|(|x|+\sqrt{2} t+K)^{\alpha} Q(t) \exp (-t) d t d x<+\infty$. 
Dealing with $h(\lambda)=n^{2} r_{n}(\lambda)$ one gets

$$
\limsup _{y \rightarrow 0^{+}}\left|\int_{\mathbb{R}} \varphi(x) r_{n}(x+i y) d x\right| \leq \frac{C}{n^{2}} .
$$

Combining (3.15) with respectively (3.12) and (3.13), one gets respectively (1.8) and (1.10).

\section{The iid case}

We consider a Hermitian matrix $X_{n}=\left[\left(X_{n}\right)_{j k}\right]_{j, k=1}^{n}$ of size $\mathrm{n}$ for which the $n^{2}$ rv $\left(\left(X_{n}\right)_{i i}\right),\left(\sqrt{2} \operatorname{Re}\left(\left(X_{n}\right)_{i j}\right)_{i<j},\left(\sqrt{2} \operatorname{Im}\left(\left(X_{n}\right)_{i j}\right)_{i<j}\right.\right.$ are independent identically distributed with common distribution $\mu / \sqrt{n}$ where $\mu$ is a symmetric distribution with variance 1 on $\mathbb{R}$ which satisfies a Poincaré inequality (see section 4.2). We call $X_{n}$ a Wigner matrix with distribution $\mu$. Let $X_{n}^{(1)}, \ldots, X_{n}^{(r)}$ be $r$ independent copies of $X_{n}$. We present our main technical tool (see [12]):

Lemma 4.1 Let $\xi$ be a real-valued rv such that $\mathbb{E}\left(|\xi|^{p+2}\right)<\infty$. Let $\phi$ be a function from $\mathbb{R}$ to $\mathbb{C}$ such that the first $p+1$ derivatives are continuous and bounded. Then,

$$
\mathbb{E}(\xi \phi(\xi))=\sum_{a=0}^{p} \frac{\kappa_{a+1}}{a !} \mathbb{E}\left(\phi^{(a)}(\xi)\right)+\epsilon
$$

where $\kappa_{a}$ are the cumulants of $\xi,|\epsilon| \leq C \sup _{t}\left|\phi^{(p+1)}(t)\right| \mathbb{E}\left(|\xi|^{p+2}\right), C$ depends on $p$ only.

In the following, we shall apply this identity with a function $\phi(\xi)$ given by the Stieljes transform of a random matrix. It follows from the Lemma 2.2 and (2.12) above that the conditions of Lemma 4.1 (bounded derivatives) are fulfilled.

\subsection{The master equation}

Note that since $\mu$ satisfies a Poincaré inequality, we have $\int|x|^{q} d \mu(x)<+\infty$ for any $q$ in $\mathbb{N}$ (see Corollary 3.2 and Proposition 1.10 in [13]). Note also that, since $\mu$ is symmetric, any odd cumulant of $\mu$ vanishes. 
Theorem 4.1 With the previous notations,

$$
\mathbb{E}\left[\sum_{i=1}^{r} a_{i} H_{n}(\lambda) a_{i} H_{n}(\lambda)+\left(a_{0}-\lambda\right) H_{n}(\lambda)+1_{m}\right]+\frac{1}{n} R_{n}(\lambda)+\epsilon_{n}=0
$$

where $\left\|\epsilon_{n}\right\| \leq \frac{P_{6}\left(\left\|\operatorname{Im}(\lambda)^{-1}\right\|\right)}{n^{2}}$ and $R_{n}(\lambda)$ denotes the quantity

$\frac{\kappa_{4}}{2} \mathbb{E}\left[\sum_{p=1}^{r} \frac{1}{n^{2}} \sum_{k, l=1}^{n} a_{p}\left(\lambda \otimes 1_{n}-S_{n}\right)_{k k}^{-1} a_{p}\left(\lambda \otimes 1_{n}-S_{n}\right)_{l l}^{-1} a_{p}\left(\lambda \otimes 1_{n}-S_{n}\right)_{k k}^{-1} a_{p}\left(\lambda \otimes 1_{n}-S_{n}\right)_{l l}^{-1}\right]$

where $\kappa_{4}$ is the fourth cumulant of the distribution $\mu$. Note that

$$
\left\|R_{n}(\lambda)\right\| \leq P_{4}\left(\left\|\operatorname{Im}(\lambda)^{-1}\right\|\right) .
$$

Proof: We shall apply formula (4.1) to the $\mathcal{M}_{m}(\mathbb{C})$-valued function $\phi(\xi)=$ $\left(\lambda \otimes 1_{n}-S_{n}\right)_{i j}^{-1}$ for $1 \leq i, j \leq n$ and $\xi$ is one of the variable $\left(X_{n}^{(p)}\right)_{k k}$, $\sqrt{2} R e\left(\left(X_{n}^{(p)}\right)_{k l}\right), \sqrt{2} \operatorname{Im}\left(\left(X_{n}^{(p)}\right)_{k l}\right)$ for $1 \leq k<l \leq n$ and $p \leq r$.

We notice that

$$
\begin{aligned}
\frac{\partial \phi}{\partial R e\left(\left(X_{n}^{(p)}\right)_{k k}\right)} & =\phi_{X_{n}^{(p)}}^{\prime} \cdot e_{k k}, 1 \leq k \leq n \\
\frac{\partial \phi}{\partial \sqrt{2} \operatorname{Re}\left(\left(X_{n}^{(p)}\right)_{k l}\right)} & =\phi_{X_{n}^{(p)}}^{\prime(e} \cdot e_{k l}, 1 \leq k<l \leq n \\
\frac{\partial \phi}{\partial \sqrt{2} \operatorname{Im}\left(\left(X_{n}^{(p)}\right)_{k l}\right)} & =\phi_{X_{n}^{(p)}}^{\prime} \cdot f_{k l}, 1 \leq k<l \leq n \\
\sqrt{2} \operatorname{Re}\left(\left(X_{n}^{(p)}\right)_{k l}\right) & =\operatorname{Tr}_{n}\left(X_{n}^{(p)} e_{k l}\right), 1 \leq k<l \leq n \\
\sqrt{2} \operatorname{Im}\left(\left(X_{n}^{(p)}\right)_{k l}\right) & =\operatorname{Tr}_{n}\left(X_{n}^{(p)} f_{k l}\right), 1 \leq k<l \leq n \\
\left(X_{n}^{(p)}\right)_{k k} & =\operatorname{Tr}_{n}\left(X_{n}^{(p)} e_{k k}\right), 1 \leq k \leq n .
\end{aligned}
$$

Let $1 \leq p \leq r, 1 \leq k \leq l \leq n$ be fixed. For simplicity, we write $\phi^{\prime}, \phi^{\prime \prime}, \phi^{\prime \prime \prime}$ for the first derivatives of $\phi$ with respect to $\sqrt{2} \operatorname{Re}\left(\left(X_{n}^{(p)}\right)_{k l}\right)$. Then, according to (2.12),

$$
\begin{aligned}
\phi^{\prime}= & {\left[\left(\lambda \otimes 1_{n}-S_{n}\right)^{-1} a_{p} \otimes e_{k l}\left(\lambda \otimes 1_{n}-S_{n}\right)^{-1}\right]_{i j} } \\
\phi^{\prime \prime}= & 2\left[\left(\lambda \otimes 1_{n}-S_{n}\right)^{-1} a_{p} \otimes e_{k l}\left(\lambda \otimes 1_{n}-S_{n}\right)^{-1} a_{p} \otimes e_{k l}\left(\lambda \otimes 1_{n}-S_{n}\right)^{-1}\right]_{i j} \\
\phi^{\prime \prime \prime}= & 6\left[\left(\lambda \otimes 1_{n}-S_{n}\right)^{-1} a_{p} \otimes e_{k l}\left(\lambda \otimes 1_{n}-S_{n}\right)^{-1} a_{p} \otimes e_{k l}\left(\lambda \otimes 1_{n}-S_{n}\right)^{-1}\right. \\
& \left.\quad a_{p} \otimes e_{k l}\left(\lambda \otimes 1_{n}-S_{n}\right)^{-1}\right]_{i j}
\end{aligned}
$$


Writing (4.1) in this setting gives

$$
\mathbb{E}\left[\operatorname{Tr}_{n}\left(X_{n}^{(p)} e_{k l}\right)\left(\lambda \otimes 1_{n}-S_{n}\right)_{i j}^{-1}\right]=\frac{1}{n} \mathbb{E}\left[\phi^{\prime}\right]+\frac{\kappa_{4}}{6 n^{2}} \mathbb{E}\left[\phi^{\prime \prime \prime}\right]+O\left(n^{-3}\right)
$$

where the $O\left(n^{-3}\right)$ means the norm of this term is smaller than $\frac{C\left\|a_{p}\right\|^{5}\left\|\operatorname{Im}(\lambda)^{-1}\right\|^{6}}{n^{3}}$. Multiplying by $n$ gives the equation, denoted by $A_{i j}^{k l}(p)$ :

$$
n \mathbb{E}\left[\operatorname{Tr}_{n}\left(X_{n}^{(p)} e_{k l}\right)\left(\lambda \otimes 1_{n}-S_{n}\right)_{i j}^{-1}\right]=\mathbb{E}\left[\phi^{\prime}\right]+\frac{\kappa_{4}}{6 n} \mathbb{E}\left[\phi^{\prime \prime \prime}\right]+O\left(n^{-2}\right)
$$

with the analogous equations with $f_{p q}$ (denoted by $\left.B_{i j}^{k l}(p)\right)$ and $e_{p p}$.

Recall how we can obtain the master equation in the gaussian case (GUE case) from (4.5) which reads in this case:

$n \mathbb{E}\left[\operatorname{Tr}_{n}\left(X_{n}^{(p)} e_{k l}\right)\left(\lambda \otimes 1_{n}-S_{n}\right)_{i j}^{-1}\right]=\mathbb{E}\left[\left(\lambda \otimes 1_{n}-S_{n}\right)^{-1} a_{p} \otimes e_{k l}\left(\lambda \otimes 1_{n}-S_{n}\right)^{-1}\right]_{i j}$.

By a linear combination with the analogous equation with $f_{k l}$, we have:

$n \mathbb{E}\left[\operatorname{Tr}_{n}\left(X_{n}^{(p)} E_{k l}\right)\left(\lambda \otimes 1_{n}-S_{n}\right)_{i j}^{-1}\right]=\mathbb{E}\left[\left(\lambda \otimes 1_{n}-S_{n}\right)^{-1} a_{p} \otimes E_{k l}\left(\lambda \otimes 1_{n}-S_{n}\right)^{-1}\right]_{i j}$

for all $1 \leq k, l \leq n$.

Now, take in the above formula $i=k, j=l$ and consider $\frac{1}{n^{2}} \sum_{k, l}$, we then obtain:

$\frac{1}{n} \sum_{k, l} \mathbb{E}\left[\left(X_{n}^{(p)}\right)_{l k}\left(\lambda \otimes 1_{n}-S_{n}\right)_{k l}^{-1}\right]=\frac{1}{n^{2}} \sum_{k, l} \mathbb{E}\left[\left(\lambda \otimes 1_{n}-S_{n}\right)_{k k}^{-1} a_{p}\left(\lambda \otimes 1_{n}-S_{n}\right)_{l l}^{-1}\right]$

that is

$$
\mathbb{E}\left[i d_{m} \otimes \operatorname{tr}_{n}\left(\left(1_{m} \otimes X_{n}^{(p)}\right)\left(\lambda \otimes 1_{n}-S_{n}\right)^{-1}\right)\right]=\mathbb{E}\left[H_{n}(\lambda) a_{p} H_{n}(\lambda)\right] .
$$

Now, from the above equation,

$$
\begin{aligned}
\mathbb{E}\left[\sum_{p=1}^{r} a_{p} H_{n}(\lambda) a_{p} H_{n}(\lambda)\right] & =\sum_{p=1}^{r} \mathbb{E}\left[i d_{m} \otimes \operatorname{tr}_{n}\left(\left(a_{p} \otimes 1_{n}\right)\left(1_{m} \otimes X_{n}^{(p)}\right)\left(\lambda \otimes 1_{n}-S_{n}\right)^{-1}\right)\right] \\
& =\sum_{p=1}^{r} \mathbb{E}\left[i d_{m} \otimes \operatorname{tr}_{n}\left(\left(a_{p} \otimes X_{n}^{(p)}\right)\left(\lambda \otimes 1_{n}-S_{n}\right)^{-1}\right)\right] \\
& \left.=\mathbb{E}\left[i d_{m} \otimes \operatorname{tr}_{n}\left(S_{n}-a_{0} \otimes 1_{n}\right)\left(\lambda \otimes 1_{n}-S_{n}\right)^{-1}\right)\right] \\
& =-1_{m}+\left(\lambda-a_{0}\right) \mathbb{E}\left[H_{n}(\lambda)\right]
\end{aligned}
$$


implying the master formula in the GUE case:

$$
\mathbb{E}\left[\sum_{p=1}^{r} a_{p} H_{n}(\lambda) a_{p} H_{n}(\lambda)+\left(a_{0}-\lambda\right) H_{n}(\lambda)+1_{m}\right]=0 .
$$

Keeping in mind these computations, we now study the terms coming from third derivatives.

We thus consider $A(p)=\frac{1}{n^{2}} \sum_{k, l} A_{k l}^{k l}(p)$ (resp. $\left.B(p)\right)$ and study all the contributions of the different terms.

\section{Study of the third derivative}

Writing as before the terms appearing in $\phi^{\prime \prime \prime}$, we can see that all the terms except one contains at least two $G_{k l}$ and then, according to Lemma 2.2. these terms will give a contribution in $O\left(n^{-2}\right)$ in $A(p)$. The only term to be considered is:

$\frac{1}{n^{2}} \sum_{k, l} \mathbb{E}\left(\left(\lambda \otimes 1_{n}-S_{n}\right)_{k k}^{-1} a_{p}\left(\lambda \otimes 1_{n}-S_{n}\right)_{l l}^{-1} a_{p}\left(\lambda \otimes 1_{n}-S_{n}\right)_{k k}^{-1} a_{p}\left(\lambda \otimes 1_{n}-S_{n}\right)_{l l}^{-1}\right)$.

Now, using the same linear combination giving (4.7) in the GUE case, we obtain that the corrective term of order $1 / n$ appearing in the iid case is:

$$
\begin{gathered}
\frac{1}{n}\left\{\frac { \kappa _ { 4 } } { 2 } \mathbb { E } \left[\frac{1}{n^{2}} \sum_{k, l=1}^{n}\left(\lambda \otimes 1_{n}-S_{n}\right)_{k k}^{-1} a_{p}\left(\lambda \otimes 1_{n}-S_{n}\right)_{l l}^{-1} a_{p}\left(\lambda \otimes 1_{n}-S_{n}\right)_{k k}^{-1}\right.\right. \\
\left.\left.a_{p}\left(\lambda \otimes 1_{n}-S_{n}\right)_{l l}^{-1}\right]\right\} .
\end{gathered}
$$

The proof of the Theorem is complete.

\subsection{Variance estimate}

We assume that $\mu$ satisfies a Poincaré inequality: there exists a positive constant $C$ such that for any $\mathcal{C}^{1}$ function $f: \mathbb{R} \rightarrow \mathbb{C}$ such that $f$ and $f^{\prime}$ are in $L^{2}(\mu)$,

$$
\mathbf{V}(f) \leq C \int\left|f^{\prime}\right|^{2} d \mu,
$$

with $\mathbf{V}(f)=\mathbb{E}\left(|f-\mathbb{E}(f)|^{2}\right)$. We refer the reader to 4 for a characterization of the measures on $\mathbb{R}$ which satisfy a Poincaré inequality (see also [1]). For example, $\mu(d x)=\exp \left(-|x|^{\alpha}\right) d x$ with $\alpha \geq 1$ satisfies the Poincaré inequality. 
For any matrices $A_{1}, \ldots, A_{r}$, define $\left\|\left(A_{1}, \ldots, A_{r}\right)\right\|_{e}^{2}:=\sum_{j=1}^{r}\left\|A_{j}\right\|_{2}^{2}$. Let $\Psi:\left(M_{n}(\mathbb{C})_{s a}\right)^{r} \rightarrow \mathbb{R}^{r n^{2}}$ be the canonical isomorphism introduced in Remark 3.4 in 9 .

Lemma 4.2 For any function $f: \mathbb{R}^{r n^{2}} \rightarrow \mathbb{C}$ be a $\mathcal{C}^{1}$-function such that $f$ and the gradient $\nabla(f)$ are both polynomially bounded,

$$
\mathbf{V}\left[f \circ \Psi\left(X_{n}^{(1)}, \ldots, X_{n}^{(r)}\right)\right] \leq \frac{C}{n} \mathbb{E}\left\{\left\|\nabla\left[f \circ \Psi\left(X^{(1)}, \ldots, X^{(r)}\right)\right]\right\|_{e}^{2}\right\} .
$$

Proof $\mu^{(n)}:=\mu / \sqrt{n}$ satisfies the Poincaré inequality

$$
\int\left|g-\int g d \mu^{(n)}\right|^{2} d \mu^{(n)} \leq \frac{C}{n} \int\left|g^{\prime}\right|^{2} d \mu^{(n)} .
$$

(4.9) readily follows by the tensorisation property of the Poincaré inequality.

\subsection{Master inequality}

We follow the lines of the proof of Theorem 4.5 in [9]. Using the master equality (4.2), we easily get

$$
\begin{gathered}
\left\|\sum_{i=1}^{r} a_{i} G_{n}(\lambda) a_{i} G_{n}(\lambda)+\left(a_{0}-\lambda\right) G_{n}(\lambda)+1_{m}+\frac{1}{n} R_{n}(\lambda)+\epsilon_{n}\right\| \\
\leq\left\|\sum_{i=1}^{r} a_{i}^{2}\right\| \mathbb{E}\left\{\left\|H_{n}(\lambda)-\mathbb{E}\left(H_{n}(\lambda)\right)\right\|^{2}\right\} .
\end{gathered}
$$

Thanks to (4.9), the following of the proof of Theorem 4.5 in [9] still holds and we similarly get

$$
\mathbb{E}\left\{\left\|H_{n}(\lambda)-E\left(H_{n}(\lambda)\right)\right\|^{2}\right\} \leq \frac{C m^{3}}{n^{2}}\left\|\sum_{i=1}^{r} a_{i}^{2}\right\|\left\|(\operatorname{Im}(\lambda))^{-1}\right\|^{4}
$$

and therefore

$$
\left\|\sum_{i=1}^{r} a_{i} G_{n}(\lambda) a_{i} G_{n}(\lambda)+\left(a_{0}-\lambda\right) G_{n}(\lambda)+1_{m}+\frac{1}{n} R_{n}(\lambda)\right\| \leq \frac{P_{6}\left(\left\|(\operatorname{Im}(\lambda))^{-1}\right\|\right)}{n^{2}} .
$$




\subsection{Estimation of $\left\|G_{n}-G\right\|$}

In the Gaussian case, Haagerup and Thorbjørnsen in [9] and Schultz in [15] prove that $G_{n}(\lambda)$ is invertible for any $\lambda$ such that $\operatorname{Im} \lambda$ is positive definite. In our more general case, we are going to use the master inequality (4.10) in order to prove that, for any $\lambda$ in some subset of $\mathcal{O}, G_{n}(\lambda)$ is invertible and to get an upper bound of $\left\|G_{n}(\lambda)^{-1}\right\|$.

Set

$$
B_{n}(\lambda)=\sum_{i=1}^{r} a_{i} G_{n}(\lambda) a_{i}+\left(a_{0}-\lambda\right)
$$

Now, from the master inequality (4.10) and (4.3), we get

$$
\left\|\sum_{i=1}^{r} a_{i} G_{n}(\lambda) a_{i} G_{n}(\lambda)+\left(a_{0}-\lambda\right) G_{n}(\lambda)+1_{m}\right\| \leq \frac{P_{6}\left(\left\|(\operatorname{Im}(\lambda))^{-1}\right\|\right)}{n} .
$$

that is

$$
\left\|B_{n}(\lambda) G_{n}(\lambda)+1_{m}\right\| \leq \frac{P_{6}\left(\left\|(\operatorname{Im}(\lambda))^{-1}\right\|\right)}{n} .
$$

Hence, for any $\lambda$ such that $\frac{P_{6}\left(\left\|(\operatorname{Im}(\lambda))^{-1}\right\|\right)}{n}<\frac{1}{2}, B_{n}(\lambda) G_{n}(\lambda)$ is invertible with

$$
\left\|\left[B_{n}(\lambda) G_{n}(\lambda)\right]^{-1}\right\| \leq 2 .
$$

Thus, for such a $\lambda, G_{n}(\lambda)$ is also obviously invertible with

$$
\left\|G_{n}(\lambda)^{-1}\right\| \leq 2\left\|B_{n}(\lambda)\right\| \leq 2\left(\left\|a_{0}\right\|+\|\lambda\|+\sum_{i=1}^{r}\left\|a_{i}\right\|^{2}\left\|(\operatorname{Im}(\lambda))^{-1}\right\|\right)
$$

Now, from the inequality (4.11) and using (4.12), we get readily that for any $\lambda$ in $\mathcal{O}$ such that $\frac{P_{6}\left(\left\|(\operatorname{Im}(\lambda))^{-1}\right\|\right)}{n}<\frac{1}{2}$,

$\left\|\sum_{i=1}^{r} a_{i} G_{n}(\lambda) a_{i}+\left(a_{0}-\lambda\right)+G_{n}(\lambda)^{-1}\right\| \leq \frac{P_{6}\left(\left\|(\operatorname{Im}(\lambda))^{-1}\right\|\right)}{n} 2\left(\left\|a_{0}\right\|+\|\lambda\|+\sum_{i=1}^{r}\left\|a_{i}\right\|^{2}\left\|(\operatorname{Im}(\lambda))^{-1}\right\|\right)$.

Define

$\mathcal{O}_{n}^{\prime}=\left\{\lambda \in \mathcal{O}, \frac{P_{6}\left(\left\|(\operatorname{Im}(\lambda))^{-1}\right\|\right)}{n} 2\left(\left\|a_{0}\right\|+\|\lambda\|+\sum_{i=1}^{r}\left\|a_{i}\right\|^{2}\left\|(\operatorname{Im}(\lambda))^{-1}\right\|\right)<\frac{1}{2\left\|(\operatorname{Im}(\lambda))^{-1}\right\|}\right\}$. 
Since $t \mapsto \frac{P_{6}\left(t^{-1}\right)}{n} 2\left(\left\|a_{0}\right\|+t+\sum_{i=1}^{r}\left\|a_{i}\right\|^{2} t^{-1}\right) t^{-1}$ is a continuous strictly decreasing function from $] 0,+\infty[$ onto $] 0,+\infty\left[\right.$, one can prove that $\mathcal{O}_{n}^{\prime}$ is an open connected subset of $M_{m}(\mathbb{C})$ by following the proof of (a) Proposition 5.6 in $[9$. Note that, using the inequality

$$
\frac{1}{\left\|(\operatorname{Im}(\lambda))^{-1}\right\|} \leq\|\lambda\|
$$

one immediately gets that for any $\lambda$ in $\mathcal{O}_{n}^{\prime}$,

$$
\left\|(\operatorname{Im}(\lambda))^{-1}\right\|\left(\left\|a_{0}\right\|+\|\lambda\|+\sum_{i=1}^{r}\left\|a_{i}\right\|^{2}\left\|(\operatorname{Im}(\lambda))^{-1}\right\|\right) \geq 1
$$

and thus that

$$
\frac{P_{6}\left(\left\|(\operatorname{Im}(\lambda))^{-1}\right\|\right)}{n} \leq \frac{1}{4} .
$$

Consequently, for any $\lambda$ in $\mathcal{O}_{n}^{\prime}, G_{n}(\lambda)$ is invertible and (4.13) holds. Defining for any $\lambda$ in $\mathcal{O}_{n}^{\prime}$,

$$
\Lambda_{n}(\lambda)=a_{0}+\sum_{i=1}^{r} a_{i} G_{n}(\lambda) a_{i}+G_{n}(\lambda)^{-1}
$$

and sticking to the proof of 9] described in section II. 2.1, we get that, for any $\lambda$ in $\mathcal{O}$,

$$
\begin{aligned}
\left\|G_{n}(\lambda)-G(\lambda)\right\| & \leq 4 \frac{P_{6}\left(\left\|(\operatorname{Im}(\lambda))^{-1}\right\|\right)}{n} 2\left(\left\|a_{0}\right\|+\|\lambda\|+\sum_{i=1}^{r}\left\|a_{i}\right\|^{2}\left\|(\operatorname{Im}(\lambda))^{-1}\right\|\right)\left\|(\operatorname{Im}(\lambda))^{-1}\right\|^{2} \\
& \leq(\|\lambda\|+K) \frac{P_{9}\left(\left\|(\operatorname{Im}(\lambda))^{-1}\right\|\right)}{n}
\end{aligned}
$$

Note that, in the following we will use (4.13) in the simplest form:

$\forall \lambda \in \mathcal{O}_{n}^{\prime}, \quad\left\|\sum_{i=1}^{r} a_{i} G_{n}(\lambda) a_{i}+\left(a_{0}-\lambda\right)+G_{n}(\lambda)^{-1}\right\| \leq(\|\lambda\|+K) \frac{P_{7}\left(\left\|(\operatorname{Im}(\lambda))^{-1}\right\|\right)}{n}$.

\subsection{Convergence of $R_{n}(\lambda)$}

Let $x_{i}, i=1, \ldots, r$ be self-adjoint operators in a $C^{*}$ probability space $(\mathcal{B}, \tau)$. We assume that the $x_{i}$ are free and identically semi-circular distributed with mean 0 and variance 1 . Then, $G$ satisfies (3.1). 
Proposition 4.1 Let a be a matrix in $M_{m}(\mathbb{C})$. Then,

$$
\mathbb{E}\left[\frac{1}{n} \sum_{k=1}^{n}\left(\lambda \otimes 1_{n}-S_{n}\right)_{k k}^{-1} a\left(\lambda \otimes 1_{n}-S_{n}\right)_{k k}^{-1}\right]=G a G+O\left(\frac{P_{10}\left(\left\|(\operatorname{Im}(\lambda))^{-1}\right\|\right)}{n}(\|\lambda\|+K)\right)
$$

Proof: We start from the resolvent identity:

$$
\lambda\left(\lambda \otimes 1_{n}-S_{n}\right)_{k k}^{-1}=1_{m}+\sum_{l=1}^{n}\left(S_{n}\right)_{k l}\left(\lambda \otimes 1_{n}-S_{n}\right)_{l k}^{-1} .
$$

We write $G^{(n)}(\lambda)=\left(\lambda \otimes 1_{n}-S_{n}\right)^{-1}$ and $D_{a}^{(n)}(\lambda)=\frac{1}{n} \sum_{k=1}^{n} G^{(n)}(\lambda)_{k k} a G^{(n)}(\lambda)_{k k}$.

From the above identity,

$$
\begin{aligned}
& \lambda \frac{1}{n} \sum_{k=1}^{n} G^{(n)}(\lambda)_{k k} a G^{(n)}(\lambda)_{k k} \\
& \quad=a \frac{1}{n} \sum_{k=1}^{n} G^{(n)}(\lambda)_{k k}+\frac{1}{n} \sum_{k, l=1}^{n}\left(S_{n}\right)_{k l} G^{(n)}(\lambda)_{l k} a G^{(n)}(\lambda)_{k k} \\
& \quad=a H_{n}(\lambda)+a_{0} D_{a}^{(n)}(\lambda)+\frac{1}{n} \sum_{p=1}^{r} a_{p} \sum_{k, l=1}^{n}\left(X_{n}^{(p)}\right)_{k l} G^{(n)}(\lambda)_{l k} a G^{(n)}(\lambda)_{k k}
\end{aligned}
$$

We take the expectation and we use the integration by part formula (4.1) for the last term:

$$
\begin{aligned}
\mathbb{E}\left(\left(X_{n}^{(p)}\right)_{k l} \Phi\left(X_{n}^{(1)}, \ldots, X_{n}^{(r)}\right)\right] & =\mathbb{E}\left(\operatorname{Tr}_{n}\left(X_{n}^{(p)} E_{l k}\right) \Phi\left(X_{n}^{(1)}, \ldots, X_{n}^{(r)}\right)\right] \\
& =\frac{1}{n} \mathbb{E}\left[\Phi_{p}^{\prime}\left(X_{n}^{(1)}, \ldots, X_{n}^{(r)}\right) \cdot E_{l k}\right]+O\left(\frac{P_{4}\left(\left\|(\operatorname{Im}(\lambda))^{-1}\right\|\right)}{n^{2}}\right)
\end{aligned}
$$

with $\Phi\left(X_{n}^{(1)}, \ldots, X_{n}^{(r)}\right)=G^{(n)}(\lambda)_{l k} a G^{(n)}(\lambda)_{k k}$. Then,

$$
\begin{aligned}
\mathbb{E}\left[\left(X_{n}^{(p)}\right)_{k l} G^{(n)}(\lambda)_{l k} a G^{(n)}(\lambda)_{k k}\right]= & \frac{1}{n} \mathbb{E}\left[G^{(n)}(\lambda)_{l l} a_{p} G^{(n)}(\lambda)_{k k} a G^{(n)}(\lambda)_{k k}\right] \\
& +\frac{1}{n} \mathbb{E}\left[G^{(n)}(\lambda)_{l k} a G^{(n)}(\lambda)_{k l} a_{p} G^{(n)}(\lambda)_{l k}\right] \\
& +O\left(\frac{P_{4}\left(\left\|(\operatorname{Im}(\lambda))^{-1}\right\|\right)}{n^{2}}\right)
\end{aligned}
$$


Thus, we obtain from the resolvent identity,

$$
\begin{aligned}
& \left(\lambda-a_{0}\right) \mathbb{E}\left(D_{a}^{(n)}(\lambda)\right)=a \mathbb{E}\left(H_{n}(\lambda)\right)+ \\
& \sum_{p=1}^{r} a_{p} \sum_{k, l=1}^{n} \frac{1}{n^{2}} \mathbb{E}\left[G^{(n)}(\lambda)_{l l} a_{p} G^{(n)}(\lambda)_{k k} a G^{(n)}(\lambda)_{k k}+\right. \\
& \left.\quad G^{(n)}(\lambda)_{l k} a G^{(n)}(\lambda)_{k l} a_{p} G^{(n)}(\lambda)_{l k}\right]+O\left(\frac{P_{4}\left(\left\|(\operatorname{Im}(\lambda))^{-1}\right\|\right)}{n}\right) .
\end{aligned}
$$

¿From Lemma 2.2.

$$
\frac{1}{n^{2}} \sum_{k, l=1}^{n} \mathbb{E}\left[G^{(n)}(\lambda)_{l k} a G^{(n)}(\lambda)_{k l} a_{p} G^{(n)}(\lambda)_{l k}\right]=O\left(\frac{P_{3}\left(\left\|(\operatorname{Im}(\lambda))^{-1}\right\|\right)}{n}\right),
$$

thus,

$$
\left(\lambda-a_{0}\right) \mathbb{E}\left(D_{a}^{(n)}(\lambda)\right)=a \mathbb{E}\left(H_{n}(\lambda)\right)+\sum_{p=1}^{r} a_{p} \mathbb{E}\left[H_{n}(\lambda) a_{p} D^{(n)}(\lambda)\right]+O\left(\frac{P_{4}\left(\left\|(\operatorname{Im}(\lambda))^{-1}\right\|\right)}{n}\right)
$$

From the estimate of the variance of $H_{n}$, we have:

$$
\mathbb{E}\left[H_{n}(\lambda) a_{p} D_{a}^{(n)}(\lambda)\right]=\mathbb{E}\left[H_{n}(\lambda)\right] a_{p} \mathbb{E}\left[D_{a}^{(n)}(\lambda)\right]+O\left(\frac{P_{4}\left(\left\|(\operatorname{Im}(\lambda))^{-1}\right\|\right)}{n}\right) .
$$

Then, using also the estimation of $\left\|G_{n}(\lambda)-G(\lambda)\right\|$ we get

$$
\left(\lambda-a_{0}-\sum_{p=1}^{r} a_{p} G(\lambda) a_{p}\right) \mathbb{E}\left(D_{a}^{(n)}(\lambda)\right)=a G+O\left(\frac{P_{9}\left(\left\|(\operatorname{Im}(\lambda))^{-1}\right\|\right)}{n}(\|\lambda\|+K)\right) .
$$

Using (3.1) and (2.9) we finally get

$$
\mathbb{E}\left(D_{a}^{(n)}(\lambda)\right)=G a G+O\left(\frac{P_{10}\left(\left\|(\operatorname{Im}(\lambda))^{-1}\right\|\right)}{n}(\|\lambda\|+K)\right) .
$$

¿From the above proposition, we obtain:

Proposition $4.2 R_{n}(\lambda)$ defined in Theorem 4.1 converges as $n$ tends to infinity to

$$
R(\lambda)=\frac{\kappa_{4}}{2} \sum_{p=1}^{r} a_{p} G(\lambda) a_{p} G(\lambda) a_{p} G(\lambda) a_{p} G(\lambda) .
$$


More precisely,

$$
\left\|R_{n}(\lambda)-R(\lambda)\right\| \leq(\|\lambda\|+K)^{2} \frac{P_{20}\left(\left\|(\operatorname{Im}(\lambda))^{-1}\right\|\right)}{n} .
$$

Proof: It's enough to prove the convergence of each coordinate of the $m \times m$ matrix $R_{n}(\lambda)$. This will actually follow from the convergence of terms of the form:

$$
\mathbb{E}\left[\alpha, \beta\left(n^{-1} \sum_{k=1}^{n} G_{k k}^{(n)} a G_{k k}^{(n)}\right){ }_{\gamma, \delta}\left(n^{-1} \sum_{k=1}^{n} G_{k k}^{(n)} b G_{k k}^{(n)}\right)\right]
$$

for $a$ et $b$ elements of the canonical basis in $\mathcal{M}_{m}(\mathbb{C})$. Since, applying Lemma 4.2, we have

$$
\mathbb{E}\left(\left\|D_{a}^{(n)}(\lambda)-\mathbb{E}\left(D_{a}^{(n)}(\lambda)\right)\right\|^{2}\right) \leq \frac{P_{6}\left(\left\|(\operatorname{Im}(\lambda))^{-1}\right\|\right)}{n},
$$

the above quantity (4.17) is of the same order as:

$$
\mathbb{E}\left[{ }_{\alpha, \beta}\left(n^{-1} \sum_{k=1}^{n} G_{k k}^{(n)} a G_{k k}^{(n)}\right)\right] \mathbb{E}\left[{ }_{\gamma, \delta}\left(n^{-1} \sum_{k=1}^{n} G_{k k}^{(n)} b G_{k k}^{(n)}\right)\right]
$$

According to Proposition 4.1, this last quantity converges towards ${ }_{\alpha, \beta}(G a G)_{\gamma, \delta}(G b G)$. Thus, the convergence of $R_{n}$ to $R$ follows with the estimation (4.16).

We define

$$
L(\lambda)=\left(i d_{m} \otimes \tau\right)\left[\left(\lambda \otimes 1_{\mathcal{B}}-s\right)^{-1}\left(R(\lambda) G(\lambda)^{-1} \otimes 1_{\mathcal{B}}\right)\left(\lambda \otimes 1_{\mathcal{B}}-s\right)^{-1}\right] .
$$

\subsection{Estimation of $\left\|G(\lambda)-G_{n}(\lambda)+\frac{1}{n} L(\lambda)\right\|$}

Following 4.24 in [15], one gets for any $\lambda$ in $\mathcal{O}_{n}^{\prime}$,

$$
\begin{aligned}
&\left\|G(\lambda)-G_{n}(\lambda)+\frac{1}{n} L(\lambda)\right\| \\
& \leq \quad\left\|\left(\lambda \otimes 1_{\mathcal{B}}-s\right)^{-1}\right\|\left\|\left(\Lambda_{n}(\lambda) \otimes 1_{\mathcal{B}}-s\right)^{-1}\right\|\left\|\Lambda_{n}(\lambda)-\lambda+\frac{1}{n} R(\lambda) G(\lambda)^{-1}\right\| \\
&+\frac{1}{n}\left\|\left(\lambda \otimes 1_{\mathcal{B}}-s\right)^{-1}\right\|\left\|R(\lambda) G(\lambda)^{-1}\right\|\left\|\left(\lambda \otimes 1_{\mathcal{B}}-s\right)^{-1}-\left(\Lambda_{n}(\lambda) \otimes 1_{\mathcal{B}}-s\right)^{-1}\right\| \\
& \leq\left\|(\operatorname{Im}(\lambda))^{-1}\right\|\left\|\left(\operatorname{Im}\left(\Lambda_{n}(\lambda)\right)\right)^{-1}\right\|\left\|\Lambda_{n}(\lambda)-\lambda+\frac{1}{n} R(\lambda) G(\lambda)^{-1}\right\| \\
&+\frac{C}{n}\left\|(\operatorname{Im}(\lambda))^{-1}\right\|^{7}\left\|\left(\operatorname{Im}\left(\Lambda_{n}(\lambda)\right)\right)^{-1}\right\|\left\|\Lambda_{n}(\lambda)-\lambda\right\|(\|\lambda\|+K)^{2}
\end{aligned}
$$


where we made use of the estimates (2.7), (2.8), (2.6) and the upper bound

$$
\|R(\lambda)\| \leq C\left\|(\operatorname{Im}(\lambda))^{-1}\right\|^{4} .
$$

Now, for any $\lambda$ in $\mathcal{O}_{n}^{\prime}$,

$$
\left\|\left(\operatorname{Im}\left(\Lambda_{n}(\lambda)\right)\right)^{-1}\right\| \leq 2\left\|(\operatorname{Im}(\lambda))^{-1}\right\|
$$

and

$$
\left\|\Lambda_{n}(\lambda)-\lambda\right\| \leq \frac{P_{6}\left(\left\|(\operatorname{Im}(\lambda))^{-1}\right\|\right)}{n}(\|\lambda\|+K) .
$$

Thus,

$$
\begin{aligned}
\left\|G(\lambda)-G_{n}(\lambda)+\frac{1}{n} L(\lambda)\right\| \leq & 2\left\|(\operatorname{Im}(\lambda))^{-1}\right\|^{2}\left\|\Lambda_{n}(\lambda)-\lambda+\frac{1}{n} R(\lambda) G(\lambda)^{-1}\right\| \\
& +\frac{P_{14}\left(\left\|(\operatorname{Im}(\lambda))^{-1}\right\|\right)}{n^{2}}(\|\lambda\|+K)^{3} .
\end{aligned}
$$

Now, for any $\lambda$ in $\mathcal{O}_{n}^{\prime}$,

$$
\begin{aligned}
\left\|\Lambda_{n}(\lambda)-\lambda+\frac{1}{n} R(\lambda) G(\lambda)^{-1}\right\| \leq & \left\|\Lambda_{n}(\lambda)-\lambda+\frac{1}{n} R_{n}(\lambda) G_{n}(\lambda)^{-1}\right\| \\
& +\frac{1}{n}\left\|R_{n}(\lambda)\left(G_{n}(\lambda)^{-1}-G(\lambda)^{-1}\right)\right\| \\
& \left.+\frac{1}{n} \|\left(R_{n}(\lambda)-R(\lambda)\right) G(\lambda)^{-1}\right) \| \\
\leq & \frac{P_{7}\left(\left\|(\operatorname{Im}(\lambda))^{-1}\right\|\right)}{n^{2}}(\|\lambda\|+K) \\
& +\frac{P_{4}\left(\left\|(\operatorname{Im}(\lambda))^{-1}\right\|\right)}{n}\left\|G_{n}(\lambda)^{-1}-G(\lambda)^{-1}\right\| \\
& +\frac{1}{n}\left\|R_{n}(\lambda)-R(\lambda)\right\|(\|\lambda\|+K)^{2}\left\|(\operatorname{Im}(\lambda))^{-1}\right\|
\end{aligned}
$$

where we used (4.10), (4.12), (4.3) and (2.8). Moreover, one easily gets

$$
\begin{aligned}
\left\|G_{n}(\lambda)^{-1}-G(\lambda)^{-1}\right\| & =\left\|G_{n}(\lambda)^{-1}\left(G(\lambda)-G_{n}(\lambda)\right) G(\lambda)^{-1}\right\| \\
& \left.\leq\left\|G_{n}(\lambda)^{-1}\right\| \| G(\lambda)-G_{n}(\lambda)\right)\|\| G(\lambda)^{-1} \| .
\end{aligned}
$$

Consequently, using the estimate (4.14) of $\left\|G_{n}(\lambda)-G(\lambda)\right\|$ together with (4.12) and (2.8), we get

$$
\left\|G_{n}(\lambda)^{-1}-G(\lambda)^{-1}\right\| \leq(\|\lambda\|+K)^{4} \frac{P_{11}\left(\left\|(\operatorname{Im}(\lambda))^{-1}\right\|\right)}{n} .
$$


We conclude that

$$
\begin{aligned}
\left\|G(\lambda)-G_{n}(\lambda)+\frac{1}{n} L(\lambda)\right\| \leq & (\|\lambda\|+K)^{4} \frac{P_{17}\left(\left\|(\operatorname{Im}(\lambda))^{-1}\right\|\right)}{n^{2}} \\
& +\frac{2}{n}\left\|R_{n}(\lambda)-R(\lambda)\right\|(\|\lambda\|+K)^{2}\left\|(\operatorname{Im}(\lambda))^{-1}\right\|^{3} .
\end{aligned}
$$

Using (4.16), we can conclude that, for any $\lambda$ in $\mathcal{O}_{n}^{\prime}$,

$$
\left\|G(\lambda)-G_{n}(\lambda)+\frac{1}{n} L(\lambda)\right\| \leq(\|\lambda\|+K)^{4} \frac{P_{23}\left(\left\|(\operatorname{Im}(\lambda))^{-1}\right\|\right)}{n^{2}} .
$$

Now, for $\lambda$ in $\mathcal{O} \backslash \mathcal{O}_{n}^{\prime}$,

$$
\begin{aligned}
1 & \left.\leq 4 \frac{P_{6}\left(\left\|(\operatorname{Im}(\lambda))^{-1}\right\|\right)}{n}\left(\left\|a_{0}\right\|+\|\lambda\|+\left\|\sum_{i=1}^{r} a_{i}^{2}\right\|\left\|(\operatorname{Im}(\lambda))^{-1}\right\|\right)\left\|(\operatorname{Im}(\lambda))^{-1}\right\|\right\} \\
& \leq(\|\lambda\|+K) \frac{P_{8}\left(\left\|(\operatorname{Im}(\lambda))^{-1}\right\|\right)}{n} .
\end{aligned}
$$

We get

$$
\begin{aligned}
\left\|G(\lambda)-G_{n}(\lambda)+\frac{1}{n} L(\lambda)\right\| \leq & \left\|G(\lambda)-G_{n}(\lambda)\right\|+\frac{1}{n}\|L(\lambda)\| \\
\leq & (\|\lambda\|+K) \frac{P_{8}\left(\left\|(\operatorname{Im}(\lambda))^{-1}\right\|\right)}{n} \\
& \times\left[(\|\lambda\|+K) \frac{P_{9}\left(\left\|(\operatorname{Im}(\lambda))^{-1}\right\|\right)}{n}+\frac{1}{n}\left\|(\operatorname{Im}(\lambda))^{-1}\right\|^{7}(\|\lambda\|+K)^{2}\right] \\
\leq & (\|\lambda\|+K)^{3} \frac{P_{17}\left(\left\|(\operatorname{Im}(\lambda))^{-1}\right\|\right)}{n^{2}} .
\end{aligned}
$$

Thus, one can easily see that one can choose $K$ and $P_{23}$ such that for any $\lambda$ in $\mathcal{O}$,

$$
\left\|G(\lambda)-G_{n}(\lambda)+\frac{1}{n} L(\lambda)\right\| \leq(\|\lambda\|+K)^{4} \frac{P_{23}\left(\left\|(\operatorname{Im}(\lambda))^{-1}\right\|\right)}{n^{2}} .
$$

Note that, since under our hypothesises, $S_{n}$ and $-S_{n}$ are identically distributed, the arguments of [15] to prove her theorem 4.5 still hold. Thus, (4.18) is also valid for any $\lambda$ such that $\operatorname{Im} \lambda$ is negative definite. 


\subsection{Spectrum of $S_{n}$}

\section{- From step 2 to step 3}

Sticking to the proof of Lemma 5.5 of [15], we get that,

$$
l(\lambda):=\operatorname{tr}_{m}\left(L\left(\lambda 1_{m}\right), \lambda \in \mathbb{C} \backslash \mathbb{R},\right.
$$

is the Stieljes transform of a distribution $\Lambda$ with compact support in $s p(s)$. Hence, the proof described in section 3.2 still holds (with $\alpha=4$ and $k=23$ ); thus we can state that for any smooth function $\varphi$ with compact support

$$
\mathbb{E}\left[\left(\operatorname{tr}_{m} \otimes \operatorname{tr}_{n}\right)\left(\varphi\left(S_{n}\right)\right)\right]=\left(\operatorname{tr}_{m} \otimes \tau\right)(\varphi(s))+\frac{1}{n} \Lambda(\varphi)+O\left(\frac{1}{n^{2}}\right)
$$

Moreover, following the proof of Lemma 5.6 in [15], one can show that $\Lambda(1)=$ 0 and deduce that, for $\varphi$ smooth, constant outside a compact set and such that $\operatorname{supp}(\varphi) \cap \operatorname{sp}(s)=\emptyset$,

$$
\mathbb{E}\left[\left(\operatorname{tr}_{m} \otimes \operatorname{tr}_{n}\right)\left(\varphi\left(S_{n}\right)\right)\right]=O\left(\frac{1}{n^{2}}\right) .
$$

\section{- Step 4}

The proof of step 4 is exactly the same as in 9 so that we have proved that, for any $\varepsilon>0$ and almost surely

$$
\operatorname{Spect}\left(S_{n}\right) \subset \operatorname{Spect}(s)+(-\varepsilon, \varepsilon)
$$

when $n$ goes to infinity. Note that this result implies that

$$
\sup _{n}\left\|X_{n}^{(p)}\right\|<+\infty \text { a.e. }
$$

\subsection{The main theorem}

\subsubsection{First inequality}

By the same arguments of [9] in Proposition 7.3, we can deduce the following inequality from the above inclusion of the spectrum of $S_{n}$.

Proposition 4.3 Almost everywhere, for all polynomials $p$ in $r$ non commuting variables,

$$
\limsup _{n \rightarrow+\infty} \| p\left(X_{n}^{(1)}, \ldots, X_{n}^{(r)}\|\leq\| p\left(x_{1}, \ldots, x_{r}\right) \| .\right.
$$




\subsubsection{Second inequality}

Proposition 4.4 Almost everywhere, for all polynomials $p$ in $r$ non commuting variables,

$$
\liminf _{n \rightarrow+\infty} \| p\left(X_{n}^{(1)}, \ldots, X_{n}^{(r)}\|\geq\| p\left(x_{1}, \ldots, x_{r}\right) \| .\right.
$$

¿From 9], Proof of Lemma 7.2, it is clear that this proposition follows from the almost sure asymptotic freeness of the $X_{n}^{(i)}$ together with the property that

$$
\text { a.e } \sup _{n}\left\|X_{n}^{(i)}\right\|<+\infty .
$$

The proof of the first point follows the proof of Theorem 6.2 in [15]; nevertheless, we modify the proof of Lemma 6.5 in [15] to get the analogue in our context without needing such a result as Lemma 6.4 in [15].

Lemma 4.3 Let d be in $\mathbb{N}^{*}, i_{1}, \ldots, i_{d}$ be in $\{1, \ldots, r\}$ and $n$ be in $\mathbb{N}^{*}$. Define $f: M_{n}(\mathbb{C})^{r} \rightarrow \mathbb{C}$ by

$$
f\left(v_{1}, \ldots, v_{r}\right)=\operatorname{tr}_{n}\left(v_{i_{1}} \ldots v_{i_{d}}\right)
$$

Then, there is a constant $C>0$ such that

$$
\mathbf{V} f\left(X_{n}^{(1)}, \ldots, X_{n}^{(r)}\right) \leq \frac{C}{n^{2}} .
$$

Proof: Applying Poincaré Inequality (4.9), we get

$$
\mathbf{V} f\left(X_{n}^{(1)}, \ldots, X_{n}^{(r)}\right) \leq \frac{C}{n} \mathbb{E}\left\{\left\|\nabla f\left(X_{n}^{(1)}, \ldots, X_{n}^{(r)}\right)\right\|_{e}^{2}\right\} .
$$

Now, let $v=\left(v_{1}, \ldots, v_{r}\right)$ be in $M_{n}(\mathbb{C})^{r}$ and $w=\left(w_{1}, \ldots, w_{r}\right)$ be in $M_{n}(\mathbb{C})_{s a}^{r}$ with $\|w\|_{e}=1$. By the Cauchy Schwartz inequality

$$
\begin{aligned}
&\left|\frac{d}{d t} t_{t=0} f(v+t w)\right|=\frac{1}{n} \mid \operatorname{Tr}_{n}\left(w_{i_{1}} v_{i_{2}} v_{i_{3}} \ldots v_{i_{d}}\right)+\operatorname{Tr}_{n}\left(v_{i_{1}} w_{i_{2}} v_{i_{3}} \ldots v_{i_{d}}\right)+\ldots \\
& \ldots+\operatorname{Tr}_{n}\left(v_{i_{1}} v_{i_{2}} \ldots v_{i_{d-1}} w_{i_{d}}\right) \mid \\
& \leq \frac{1}{n}\left\{\left\|w_{i_{1}}\right\|\left\|_{2}\right\| v_{i_{2}} v_{i_{3}} \ldots v_{i_{d}}\left\|_{2}+\ldots+\right\| w_{i_{d}}\|\|_{2}\left\|v_{i_{1}} v_{i_{2}} \ldots v_{i_{d-1}}\right\|_{2}\right\} \\
& \leq \frac{1}{n}\left\{\left\|v_{i_{2}} v_{i_{3}} \ldots v_{i_{d}}\right\|_{2}+\left\|v_{i_{1}} v_{i_{3}} \ldots v_{i_{d}}\right\|_{2}+\ldots+\left\|v_{i_{1}} v_{i_{2}} \ldots v_{i_{d-1}}\right\|_{2}\right\}
\end{aligned}
$$


Thus,

$$
\begin{aligned}
\left\|\nabla f\left(X_{n}^{(1)}, \ldots, X_{n}^{(r)}\right)\right\|_{e}^{2} \leq \frac{1}{n^{2}}[ & \left\{\operatorname{Tr}_{n}\left(X_{n}^{\left(i_{2}\right)} X_{n}^{\left(i_{3}\right)} \ldots X_{n}^{\left(i_{d}\right)} X_{n}^{\left(i_{d}\right)} \ldots X_{n}^{\left(i_{2}\right)}\right)\right\}^{\frac{1}{2}} \\
& \left.+\ldots+\left\{\operatorname{Tr}_{n}\left(X_{n}^{\left(i_{1}\right)} \ldots X_{n}^{\left(i_{d-1}\right)} X_{n}^{\left(i_{d-1}\right)} \ldots X_{n}^{\left(i_{1}\right)}\right)\right\}^{\frac{1}{2}}\right]^{2} \\
\leq \frac{C}{n^{2}}\{ & \operatorname{Tr}_{n}\left(X_{n}^{\left(i_{2}\right)} X_{n}^{\left(i_{3}\right)} \ldots X_{n}^{\left(i_{d}\right)} X_{n}^{\left(i_{d}\right)} \ldots X_{n}^{\left(i_{2}\right)}\right) \\
& \left.+\ldots+\operatorname{Tr}_{n}\left(X_{n}^{\left(i_{1}\right)} \ldots X_{n}^{\left(i_{d-1}\right)} X_{n}^{\left(i_{d-1}\right)} \ldots X_{n}^{\left(i_{1}\right)}\right)\right\}
\end{aligned}
$$

for some constant $C$ depending on $d$, and we get that

$$
\begin{aligned}
\mathbb{E}\left(\left\|\nabla f\left(X_{n}^{(1)}, \ldots, X_{n}^{(r)}\right)\right\|_{e}^{2}\right) \leq \frac{C}{n}\left\{\mathbb{E}\left(\operatorname{tr}_{n}\left(X_{n}^{\left(i_{2}\right)} X_{n}^{\left(i_{3}\right)} \ldots X_{n}^{\left(i_{d}\right)} X_{n}^{\left(i_{d}\right)} \ldots X_{n}^{\left(i_{2}\right)}\right)\right)\right. \\
\\
\left.\quad+\ldots+\mathbb{E}\left(\operatorname{tr}_{n}\left(X_{n}^{\left(i_{1}\right)} \ldots X_{n}^{\left(i_{d-1}\right)} X_{n}^{\left(i_{d-1}\right)} \ldots X_{n}^{\left(i_{1}\right)}\right)\right)\right\}
\end{aligned}
$$

Each term inside the brackets of the left hand side is uniformly bounded in $n$ since it converges as $n$ tends to infinity according to the result of asymptotic freeness in mean of Dykema in [6]. The result follows.

Lemma 4.3 yields the almost sure asymptotic freeness of the $X_{n}^{(i)}$ using the Borel Cantelli lemma.

In conclusion,

Theorem 4.2 Let $X_{n}^{(1)}, \ldots, X_{n}^{(r)}$ be independent Wigner matrices associated to a symmetric distribution $\mu$ which satisfies a Poincaré inequality. Let $\left(x_{1}, \ldots, x_{r}\right)$ be a semicircular system. Then, almost everywhere, for all polynomials $p$ in $r$ non commuting variables

$$
\lim _{n \rightarrow+\infty}\left\|p\left(X_{n}^{(1)}, \ldots, X_{n}^{(r)}\right)\right\|=\left\|p\left(x_{1}, \ldots, x_{r}\right)\right\| .
$$

\section{The Wishart case}

We consider a $n \times n$ Hermitian matrix $Y$, distributed as a Wishart matrix of parameter $p(n) \geq n$ and variance $\frac{1}{n}$ that is with density w.r.t the Lebesgue measure $d M$ on $\mathcal{M}_{s a}(\mathbb{C})$ :

$$
C_{n, p} 1_{(M \geq 0)}(\operatorname{det}(M))^{p(n)-n} \exp (-n \operatorname{tr}(M)) .
$$


We assume that $\frac{p(n)}{n} \underset{n \rightarrow \infty}{\longrightarrow} \alpha$ for some $\alpha \geq 1$. More precisely, according to Dirichlet theorem ([16], Lemme 14.1), there exists subsequences $p(n)$ and $q(n)$ of integers tending to $\infty$ such that:

$$
\left|\frac{p(n)}{q(n)}-\alpha\right| \leq \frac{1}{q(n)^{2}} .
$$

So, we shall consider a matrix $Y$ of size $q(n)$ and parameter $p(n)$. For simplicity, we shall denote the subsequence $q(n)$ by $n$ and therefore, we will assume in this section that:

$$
\left|\frac{p(n)}{n}-\alpha\right| \leq \frac{1}{n^{2}} .
$$

It is well know that the spectral measure of $Y$ converges to the so called Marchenko-Pastur distribution $\mu_{\alpha}$ [14]:

$$
\mu_{\alpha}(d x)=\frac{\sqrt{\left((\sqrt{\alpha}+1)^{2}-x\right)\left(x-(\sqrt{\alpha}-1)^{2}\right)}}{2 \pi x} 1_{\left[(\sqrt{\alpha}-1)^{2},(\sqrt{\alpha}+1)^{2}\right]}(x) d x .
$$

\subsection{Differentiation formula for the Wishart ensemble}

Lemma 5.1 Let $\Phi$ a $C^{1}$ function on $\mathcal{M}_{s a}(\mathbb{C})$ with $\Phi(0)=0$, then:

$$
\mathbb{E}\left[\Phi^{\prime}(Y) \cdot H\right]-n \mathbb{E}\left[\Phi(Y) \operatorname{Tr}_{n}(H)\right]+(p(n)-n) \mathbb{E}\left[\Phi(Y) \operatorname{Tr}_{n}\left(Y^{-1} H\right)\right]=0
$$

for all hermitian matrix $H$, or by linearity for $H=E_{j k}, 1 \leq j, k \leq n$.

Proof: Since the Lebesgue measure is invariant by translation,

$\mathbb{E}[\Phi(Y)]=\int \Phi(M+\epsilon X) \exp \left(-n \operatorname{Tr}_{n}(M+\epsilon X)\right)(\operatorname{det}(M+\epsilon X))^{p(n)-n} 1_{(M+\epsilon X \geq 0)} d M$.

Now, by differentiation with respect to $\epsilon$ and taking $\epsilon=0$, we obtain (5.2) using $\partial(\operatorname{det} M)^{s}=s(\operatorname{det} M)^{s} M^{-1}$.

\subsection{The master equation}

Let $\left(X_{n}^{(1)}, \ldots, X_{n}^{(r)}\right)_{i=1, \ldots r}$ be $r$ independent copies of the random matrix $Y$. We shall apply (5.2) with

$$
\Phi\left(X_{n}^{(l)}\right)=\left[\left(1_{m} \otimes X_{n}^{(l)}\right)\left(\lambda \otimes 1_{n}-S_{n}\right)^{-1}\right]_{j k} \in \mathcal{M}_{m}(\mathbb{C})
$$


and $H=E_{j k}$. Then,

$\Phi^{\prime}\left(X_{n}^{(l)}\right) \cdot E_{j k}=\left(\lambda \otimes 1_{n}-S_{n}\right)_{k k}^{-1}+\left[\left(1_{m} \otimes X_{n}^{(l)}\right)\left(\lambda \otimes 1_{n}-S_{n}\right)^{-1}\right]_{j j} a_{l}\left[\left(\lambda \otimes 1_{n}-S_{n}\right)^{-1}\right]_{k k}$

and

$$
\Phi\left(X_{n}^{(l)}\right) \operatorname{Tr}_{n}\left(\left(X_{n}^{(l)}\right)^{-1} E_{j k}\right)=\left(X_{n}^{(l)}\right)_{k j}^{-1}\left[\left(1_{m} \otimes X_{n}^{(l)}\right)\left(\lambda \otimes 1_{n}-S_{n}\right)^{-1}\right]_{j k} .
$$

The sum over $j$ of the terms in the above equation gives:

$$
\left(\lambda \otimes 1_{n}-S_{n}\right)_{k k}^{-1} .
$$

Now, if we sum the identities obtained by (5.2) over $j, k$, and dividing by $n^{2}$, we obtain:

$$
\begin{aligned}
& \mathbb{E}\left[i d \otimes \operatorname{tr}_{n}\left(\left(\lambda \otimes 1_{n}-S_{n}\right)^{-1}\right)\right] \\
& \quad+\mathbb{E}\left[i d \otimes \operatorname{tr}_{n}\left(\left(1_{m} \otimes X_{n}^{(l)}\right)\left(\lambda \otimes 1_{n}-S_{n}\right)^{-1}\right) a_{l} i d \otimes \operatorname{tr}_{n}\left(\left(\lambda \otimes 1_{n}-S_{n}\right)^{-1}\right)\right] \\
& \quad-\mathbb{E}\left[i d \otimes \operatorname{tr}_{n}\left(\left(1_{m} \otimes X_{n}^{(l)}\right)\left(\lambda \otimes 1_{n}-S_{n}\right)^{-1}\right)\right] \\
& \quad+\left(\frac{p(n)}{n}-1\right) \mathbb{E}\left[i d \otimes \operatorname{tr}_{n}\left(\left(\lambda \otimes 1_{n}-S_{n}\right)^{-1}\right)\right]=0
\end{aligned}
$$

which can be written as:

$$
\begin{gathered}
\mathbb{E}\left[i d \otimes \operatorname{tr}_{n}\left(\left(1_{m} \otimes X_{n}^{(l)}\right)\left(\lambda \otimes 1_{n}-S_{n}\right)^{-1}\right)\left(1_{m}-a_{l} i d \otimes \operatorname{tr}_{n}\left(\left(\lambda \otimes 1_{n}-S_{n}\right)^{-1}\right)\right]\right. \\
=\frac{p(n)}{n} \mathbb{E}\left[i d \otimes \operatorname{tr}_{n}\left(\left(\lambda \otimes 1_{n}-S_{n}\right)^{-1}\right)\right] .
\end{gathered}
$$

Proposition 5.1 1. For $\lambda \in \mathcal{O}$, $\mid \mathbb{E}\left[i d \otimes \operatorname{tr}_{n}\left(\left(1_{m} \otimes X_{n}^{(l)}\right)\left(\lambda \otimes 1_{n}-S_{n}\right)^{-1}\right)\left(1_{m}-a_{l} i d \otimes \operatorname{tr}_{n}\left(\left(\lambda \otimes 1_{n}-S_{n}\right)^{-1}\right)\right]-\right.$ $\mathbb{E}\left[i d \otimes \operatorname{tr}_{n}\left(\left(1_{m} \otimes X_{n}^{(l)}\right)\left(\lambda \otimes 1_{n}-S_{n}\right)^{-1}\right)\right] \mathbb{E}\left[\left(1_{m}-a_{l} i d \otimes \operatorname{tr}_{n}\left(\left(\lambda \otimes 1_{n}-S_{n}\right)^{-1}\right)\right] \mid\right.$ $\leq \frac{P_{4}\left(\left\|(\operatorname{Im}(\lambda))^{-1}\right\|\right)}{n^{2}}$.

2. For $\lambda \in \mathcal{O}$,

$$
\left\|G_{n}(\lambda)^{-1}\right\| \leq(\|\lambda\|+K)^{2}\left\|(\operatorname{Im}(\lambda))^{-1}\right\|
$$


3. If $a_{l}$ is invertible and $\lambda \in \mathcal{O}$, then $\left(1_{m}-a_{l} G_{n}(\lambda)\right)$ is invertible and

$$
\begin{gathered}
\left\|\left(1_{m}-a_{l} G_{n}(\lambda)\right)^{-1}\right\| \leq\left\|a_{l}^{-1}\right\|(\|\lambda\|+K)^{2}\left\|(\operatorname{Im}(\lambda))^{-1}\right\| . \\
\text { If }\left\|(\operatorname{Im}(\lambda))^{-1}\right\|<\frac{1}{2\left\|a_{l}\right\|}, \text { then }\left(1_{m}-a_{l} G_{n}(\lambda)\right) \text { is invertible and } \\
\left\|\left(1_{m}-a_{l} G_{n}(\lambda)\right)^{-1}\right\| \leq 2 .
\end{gathered}
$$

\section{Sketch of Proof:}

1. The variance estimate follows from the Gaussian Poincaré inequality since we can write $Y=\frac{1}{n} X^{*} X$ for a rectangular Gaussian matrix $X$. We proceed as in [9, Section 4]. We need some estimate on the maximal eigenvalue of $Y$, i.e. $\mathbb{E}\left[\lambda_{\text {max }}\right]$ and $\mathbb{E}\left[\lambda_{\text {max }}^{3}\right]$ are bounded, independently of $n$. This can be proved, as in Lemma 5.1 of [9], using previous results in [8].

2. The proof is the same as Proposition 5.2 in [9].

3. If $a_{l}$ is invertible,

$$
\left(1-a_{l} G_{n}(\lambda)\right)=a_{l}\left(a_{l}^{-1}-G_{n}(\lambda)\right):=a_{l} T .
$$

Now, the matrix $T$ satisfies, $\operatorname{Im}(T)=-\operatorname{Im}\left(G_{n}(\lambda)\right)$ and thus is positive definite (see the proof of Proposition 5.2 in [9]). Its inverse $T^{-1}$ satisfies:

$$
\left\|T^{-1}\right\| \leq\left\|\operatorname{Im}(T)^{-1}\right\|=\left\|\operatorname{Im}\left(G_{n}(\lambda)\right)^{-1}\right\| \leq(\|\lambda\|+K)^{2}\left\|(\operatorname{Im}(\lambda))^{-1}\right\| .
$$

(5.5) follows from the majoration $\left\|G_{n}(\lambda)\right\| \leq\left\|(\operatorname{Im}(\lambda))^{-1}\right\|$.

From (5.4) and Proposition 5.1, we obtain:

$$
\begin{aligned}
& \| \sum_{l=1}^{r} a_{l} \mathbb{E}\left[i d \otimes \operatorname{tr}_{n}\left(\left(1_{m} \otimes Y^{(l)}\right)\left(\lambda \otimes 1_{n}-S_{n}\right)^{-1}\right)\right]- \\
& \quad \frac{p(n)}{n} \sum_{l=1}^{r} a_{l} \mathbb{E}\left[H_{n}(\lambda)\right]\left(1_{m}-a_{l} \mathbb{E}\left[H_{n}(\lambda)\right]\right)^{-1} \| \leq(\|\lambda\|+K)^{2} \frac{P_{5}\left(\left\|(\operatorname{Im}(\lambda))^{-1}\right\|\right)}{n^{2}}
\end{aligned}
$$

The first line of the above equation equals:

$$
\left.\mathbb{E}\left[i d \otimes \operatorname{tr}_{n}\left(S_{n}-a_{0} \otimes 1_{n}\right)\left(\lambda \otimes 1_{n}-S_{n}\right)^{-1}\right)\right]=-1_{m}+\left(\lambda-a_{0}\right) \mathbb{E}\left[H_{n}(\lambda)\right] .
$$

We have thus obtain the master inequality:

$$
\left\|-a_{0} G_{n}(\lambda)+\lambda G_{n}(\lambda)-\frac{p(n)}{n} \sum_{l=1}^{r} a_{l} G_{n}(\lambda)\left(1_{m}-a_{l} G_{n}(\lambda)\right)^{-1}-1_{m}\right\| \leq(\|\lambda\|+K)^{2} \frac{P_{5}\left(\left\|(\operatorname{Im}(\lambda))^{-1}\right\|\right)}{n^{2}}
$$


or since the matrices $\left(1_{m}-a_{l} G_{n}(\lambda)\right)^{-1}$ and $a_{l} G_{n}(\lambda)$ commute,

$\left\|-a_{0} G_{n}(\lambda)+\lambda G_{n}(\lambda)-\frac{p(n)}{n} \sum_{l=1}^{r}\left(1_{m}-a_{l} G_{n}(\lambda)\right)^{-1} a_{l} G_{n}(\lambda)-1_{m}\right\| \leq(\|\lambda\|+K)^{2} \frac{P_{5}\left(\left\|(\operatorname{Im}(\lambda))^{-1}\right\|\right)}{n^{2}}$

\subsection{Estimation of $\left\|G_{n}(\lambda)-G(\lambda)\right\|$}

Let $x_{i}, i \leq r$ be a free family of self adjoint variables in a $C^{*}$-probability space $(\mathcal{B}, \tau)$, with Marchenko-Pastur distribution $\mu_{\alpha}$, with parameter $\alpha$.

$$
s=a_{0} \otimes 1_{\mathcal{B}}+\sum_{i=1}^{r} a_{i} \otimes x_{i}
$$

Using the known expression of the $R$ transform of the distribution of $x_{i}$ (see [5], [10, Example 3.3.5 $\left.]^{2}\right)$ :

$$
R_{x}(z)=\alpha(1-z)^{-1}, z \in \mathbb{C} \backslash \mathbb{R}
$$

we can show the following

Lemma 5.2 G satisfies the following equation: for $\lambda \in \mathcal{O}$,

$$
a_{0}+\alpha \sum_{i=1}^{r}\left(1_{m}-a_{i} G(\lambda)\right)^{-1} a_{i}+G(\lambda)^{-1}=\lambda
$$

Sketch of Proof: From the definition of the $R$ transformation with amalgation over $M_{m}(\mathbb{C})$, we can show that:

$$
R_{a \otimes x}(\lambda)=R_{x}(a \lambda) a, a \in M_{m}(\mathbb{C})_{s a}, \lambda \in \mathcal{O}
$$

and then, by freeness asumption

$$
R_{\sum_{i=1}^{r} a_{i} \otimes x_{i}}(\lambda)=\sum_{i=1}^{r} R_{x_{i}}\left(a_{i} \lambda\right) a_{i}
$$

(5.8) follows, using the relation between $R$ and $G$.

\footnotetext{
${ }^{2}$ We warm the reader that the $R$ transform defined in this book differs by a factor $z$ from the Voiculescu $R$ transform we used here
} 
Theorem 5.1 For any $\lambda \in \mathcal{O}$,

$$
\left\|G(\lambda)-G_{n}(\lambda)\right\| \leq(\|\lambda\|+K)^{4} \frac{P_{8}\left(\left\|(\operatorname{Im}(\lambda))^{-1}\right\|\right)}{n^{2}} .
$$

Proof: We can proceed as in the proof of Theorem 5.7 in 9]. We just mention the different steps:

Step 1: Define $\Lambda_{n}(\lambda)=a_{0}+G_{n}(\lambda)^{-1}+\alpha \sum_{l}\left(1_{m}-a_{l} G_{n}(\lambda)\right)^{-1} a_{l}$. From the master inequality (5.7), Proposition [5.1 and (5.1), we can show that:

$$
\left\|\lambda-\Lambda_{n}(\lambda)\right\| \leq(\|\lambda\|+K)^{4} \frac{P_{6}\left(\left\|(\operatorname{Im}(\lambda))^{-1}\right\|\right)}{n^{2}} .
$$

Then, for $\lambda \in \mathcal{O}_{n}^{\prime}$ of the form

$$
\mathcal{O}_{n}^{\prime}=\left\{\lambda \in \mathcal{O},(\|\lambda\|+K)^{4} \frac{P_{6}\left(\left\|(\operatorname{Im}(\lambda))^{-1}\right\|\right)}{n^{2}}<\frac{1}{2} \frac{1}{\left\|(\operatorname{Im}(\lambda))^{-1}\right\|}\right\},
$$

we have $\operatorname{Im}\left(\Lambda_{n}(\lambda)\right) \geq \frac{1}{2\left\|(\operatorname{Im}(\lambda))^{-1}\right\|}$ and in particular $\Lambda_{n}(\lambda) \in \mathcal{O}$.

Step 2: For $\lambda \in \mathcal{O}_{n}^{\prime}$, we can consider $G\left(\Lambda_{n}(\lambda)\right)$ and we have, from the identity (5.8):

$a_{0}+G_{n}(\lambda)^{-1}+\alpha \sum_{l}\left(1_{m}-a_{l} G_{n}(\lambda)\right)^{-1} a_{l}=a_{0}+G\left(\Lambda_{n}(\lambda)\right)^{-1}+\alpha \sum_{l}\left(1_{m}-a_{l} G\left(\Lambda_{n}(\lambda)\right)\right)^{-1} a_{l}$.

Lemma 5.3 (see [9], Propostion 5.6) For $\lambda \in \mathcal{O}_{n}^{\prime}$,

$$
G\left(\Lambda_{n}(\lambda)\right)=G_{n}(\lambda) .
$$

Proof: As in [9, it's enough to prove (5.11) for $\lambda \in \mathcal{O}_{n}^{\prime \prime}$, a non empty subset of the connected subset $\mathcal{O}_{n}^{\prime}$. Put $x=G_{n}(\lambda)$ and $y=G\left(\Lambda_{n}(\lambda)\right)$, then, from (5.10),

$$
a_{0}+x^{-1}+\alpha \sum_{l}\left(1_{m}-a_{l} x\right)^{-1} a_{l}=a_{0}+y^{-1}+\alpha \sum_{l}\left(1_{m}-a_{l} y\right)^{-1} a_{l}
$$

so that

$$
y+\alpha \sum_{l} x\left(1_{m}-a_{l} x\right)^{-1} a_{l} y=x+\alpha \sum_{l} x\left(1_{m}-a_{l} y\right)^{-1} a_{l} y,
$$


Thus,

$$
\begin{aligned}
y-x & =\alpha \sum_{l} x\left[\left(1_{m}-a_{l} y\right)^{-1}-\left(1_{m}-a_{l} x\right)^{-1}\right] a_{l} y \\
& =\alpha \sum_{l} x\left(1_{m}-a_{l} y\right)^{-1}\left[\left(1-a_{l} x\right)-\left(1-a_{l} y\right)\right]\left(1_{m}-a_{l} x\right)^{-1} a_{l} y \\
& =\alpha \sum_{l} x\left(1_{m}-a_{l} y\right)^{-1} a_{l}(y-x)\left(1_{m}-a_{l} x\right)^{-1} a_{l} y
\end{aligned}
$$

In particular, we have,

$$
\|y-x\| \leq\left(\alpha\|x\|\|y\| \sum_{l}\left\|\left(1_{m}-a_{l} y\right)^{-1}\right\|\left\|\left(1_{m}-a_{l} x\right)^{-1}\right\|\left\|a_{l}\right\|^{2}\right)\|y-x\|
$$

Now, we have

$$
\|x\|=\left\|G_{n}(\lambda)\right\| \leq\left\|(\operatorname{Im}(\lambda))^{-1}\right\|
$$

and

$$
\|y\|=\left\|G\left(\Lambda_{n}(\lambda)\right)\right\| \leq\left(\left\|\left(\operatorname{Im}\left(\Lambda_{n}(\lambda)\right)\right)^{-1}\right\| \leq 2\left\|(\operatorname{Im}(\lambda))^{-1}\right\|\right.
$$

for $\lambda \in \mathcal{O}_{n}^{\prime}$ (see Step 1).

Moreover, from Proposition 5.1. for $\left\|(\operatorname{Im}(\lambda))^{-1}\right\|$ small enough,

$$
\left\|\left(1_{m}-a_{l} x\right)^{-1}\right\| \leq 2 ;\left\|\left(1_{m}-a_{l} y\right)^{-1}\right\| \leq 2 .
$$

Set

$\mathcal{O}_{n}^{\prime \prime}=\left\{\lambda \in \mathcal{O}_{n}^{\prime} ; \alpha\left\|G_{n}(\lambda)\right\|\left\|G\left(\Lambda_{n}(\lambda)\right)\right\| \sum_{l}\left\|\left(1_{m}-a_{l} G\left(\Lambda_{n}(\lambda)\right)\right)^{-1}\right\|\left\|\left(1_{m}-a_{l} G_{n}(\lambda)\right)^{-1}\right\|\left\|a_{l}\right\|^{2}<1\right\}$,

Then, from (5.12), for $\lambda \in \mathcal{O}_{n}^{\prime \prime}, G\left(\Lambda_{n}(\lambda)\right)=G_{n}(\lambda)$. Now, it is easy to see, from the above estimates, that $\lambda=i t 1_{m} \in \mathcal{O}_{n}^{\prime \prime}$ for $t$ large enough, so $\mathcal{O}_{n}^{\prime \prime}$ is a non empty set.

Step 3: The estimation of $G\left(\Lambda_{n}(\lambda)\right)-G(\lambda)$ is obtained as in Subsection 3.1 (considering the two cases $\lambda \in \mathcal{O}_{n}^{\prime}$ and $\lambda \in \mathcal{O} \backslash \mathcal{O}_{n}^{\prime}$ ).

\subsection{The spectrum of $S_{n}$}

¿From Theorem 5.1 and the proof described in Section 3.2 (see also Section 6 in [9]), we can prove that for $\varphi$ smooth, constant outside a compact set 
and such that $\operatorname{supp}(\varphi) \cap s p(s)=\emptyset$

$$
\mathbb{E}\left[\left(\operatorname{tr}_{m} \otimes \operatorname{tr}_{n}\right)\left(\varphi\left(S_{n}\right)\right)\right]=O\left(\frac{1}{n^{2}}\right) .
$$

from which we deduce that, for any $\varepsilon>0$ and almost surely

$$
\operatorname{Spect}\left(S_{n}\right) \subset \operatorname{Spect}(s)+(-\varepsilon, \varepsilon)
$$

when $n$ goes to infinity.

\subsection{The main theorem}

We can now prove:

Theorem 5.2 There exists a set $N$ of probability 0 such that for all non commutative polynomial $p$ in $r$ variables, and all $\omega \in \Omega \backslash N$,

$$
\lim _{n \longrightarrow \infty}\left\|p\left(X_{n}^{(1)}(\omega), \ldots, X_{n}^{(r)}(\omega)\right)\right\|=\left\|p\left(x_{1}, \ldots x_{r}\right)\right\| .
$$

Proof: The inequality

$$
\limsup _{n \rightarrow \infty}\left\|p\left(X_{n}^{(1)}, \ldots, X_{n}^{(r)}\right)\right\| \leq\left\|p\left(x_{1}, \ldots x_{r}\right)\right\| \text { a.s. }
$$

follows from the above inclusion of the spectrum of $S_{n}$ and the arguments developed in [9], Section 7. The reverse inequality

$$
\liminf _{n \longrightarrow \infty}\left\|p\left(X_{n}^{(1)}, \ldots, X_{n}^{(r)}\right)\right\| \geq\left\|p\left(x_{1}, \ldots x_{r}\right)\right\| \text { a.s. }
$$

follows, as in Lemma 7.2 in [9], from the a.s. asymptotic freeness of the $\left(X_{n}^{(i)}\right)_{i=1, \ldots, r}$ and $\sup _{n}\left\|X_{n}^{(i)}\right\|<\infty$ a.s.. The first point was proved by Hiai and Petz (see [10, [11]) and the second point follows from (5.14).

Remark: If we only assume the convergence of $\frac{p(n)}{n}$ to $\alpha$ with $\left|\frac{p(n)}{n}-\alpha\right| \leq \frac{1}{n}$, then an extra term appears in the estimation of $G-G_{n}$ at order $n^{-2}$, namely:

$$
\left\|G(\lambda)-G_{n}(\lambda)+\left(\alpha-\frac{p(n)}{n}\right) L(\lambda)\right\| \leq \frac{C(\lambda)}{n^{2}}
$$

with

$$
L(\lambda)=\left(i d_{m} \otimes \tau\right)\left[\left(\lambda \otimes 1_{\mathcal{B}}-s\right)^{-1}\left(R(\lambda) \otimes 1_{\mathcal{B}}\right)\left(\lambda \otimes 1_{\mathcal{B}}-s\right)^{-1}\right]
$$

and $R(\lambda)=\sum_{l}\left(1_{m}-a_{l} G(\lambda)\right)^{-1} a_{l}$.

As in Schultz [15] and in the iid case (see Section 4), this term gives rise to a distribution with compact support in $s p(s)$ and the conclusion remains true. 


\section{References}

[1] Ané, C., Blachère, S., Chafaï, D., Fougères, P., Gentil, I., Malrieu, F., Roberto, C., Scheffer, G. Sur les inégalités de Sobolev logarithmiques. (French) [Logarithmic Sobolev inequalities] Panoramas et Synthèses [Panoramas and Syntheses], 10. Société Mathématique de France, Paris, 2000.

[2] Bai, Z. D.: Methodology in Spectral analysis of large dimensional random matrices. A review. Statistica Sinica 9 (1999), pp 611-677.

[3] Bai, Z.D., Yin, Y. Q.: Necessary and sufficient conditions for almost sure convergence of the largest eigenvalue of a Wigner matrix. Ann. of Proba. 16 (1988), pp 1729-1741.

[4] Bobkov, S., Gotze.: Exponential integrability and transportation cost related to logarithmic Sobolev inequalities. J. Funct. Anal. 163 (1999), pp 1-28.

[5] Capitaine, M. and Casalis, M.:Asymptotic freeness by generalized moments for Gaussian and Wishart matrices. Application to beta random matrices. Indiana Univ. Math. J. 53 (2004), pp 397-431.

[6] Dykema, K.: On Certain Free Product Factors via an Extended Matrix Model. J. Funct. Anal. 112 (1993), pp 31-60.

[7] Geman S.: A limit theorem for the norm of random matrices. Annals of Probability 8 (1980) pp 252-261.

[8] Haagerup, U. and Thorbjørnsen, S.: Random matrices with complex Gaussian entries. Expo. Math. 21 (2003), pp 293-337.

[9] Haagerup, U. and Thorbjørnsen, S.: A new application of random matrices: $\operatorname{Ext}\left(C_{\text {red }}^{*}\left(F_{2}\right)\right)$ is not a group. To appear in Ann. Math.

[10] Hiai, F. and Petz, D.: "The semicircle law, free random variables and entropy." Mathematical Surveys and Monographs, 77. American Mathematical Society, Providence, RI, 2000.

[11] Hiai, F. and Petz, D.: Asymptotic freeness almost everywhere for random matrices. Acta Sci. Math. 66 (2000) pp 809-834. 
[12] Khorunzhy, A., Khoruzhenko, B., Pastur,L.: Asymptotic properties of large random matrices with independent entries, J. Math. Phys. 37 (1996), pp 5033-5060.

[13] Ledoux, L.: "The Concentration of Measure Phenomenon." Mathematical Surveys and Monographs, Volume 89, A.M.S, 2001.

[14] Marchenko, V. and Pastur, L.: The distribution of eigenvalues in a certain sets of random matrices. Math. Sb. 72 (1967), pp 507-536.

[15] Schultz, H.: Non-commutative polynomials of independent Gaussian random matrices. The real and symplectic cases. To appear in Prob. Th. Rel. Fields.

[16] Tenenbaum, G.: "Introduction à la théorie analytique et probabiliste des nombres." Institut Elie Cartan, Nancy, 1990.

[17] Thorbjørnsen, S.: Mixed moments of Voiculescu's Gaussian Random matrices. J. Funct. Anal. 176 (2000), pp 213-246.

[18] Voiculescu, D.: Limit laws for random matrices and free products. Invent. Math. 104 (1991), pp 201-220. 